INT-PUB-11-007

\title{
Electric properties of the Beryllium-11 system in Halo EFT
}

\author{
H.-W. Hammer* \\ Helmholtz-Institut für Strahlen- und Kernphysik \\ (Theorie) and Bethe Center for Theoretical Physics, \\ Universität Bonn, 53115 Bonn, Germany \\ D. R. Phillips $\overbrace{}^{\dagger}$ \\ Department of Physics and Astronomy, \\ Ohio University, Athens, Ohio 45701, USA and \\ Helmholtz-Institut für Strahlen- und Kernphysik \\ (Theorie) and Bethe Center for Theoretical Physics, \\ Universität Bonn, 53115 Bonn, Germany
}

\begin{abstract}
We compute E1 transitions and electric radii in the Beryllium-11 nucleus using an effective field theory that exploits the separation of scales in this halo system. We fix the leading-order parameters of the EFT from measured data on the $1 / 2^{+}$and $1 / 2^{-}$levels in ${ }^{11} \mathrm{Be}$ and the $\mathrm{B}(\mathrm{E} 1)$ strength for the transition between them. We then obtain predictions for the $\mathrm{B}(\mathrm{E} 1)$ strength for Coulomb dissociation of the ${ }^{11} \mathrm{Be}$ nucleus to the continuum. We also compute the charge radii of the $1 / 2^{+}$and $1 / 2^{-}$states. Agreement with experiment within the expected accuracy of a leadingorder computation in this EFT is obtained. We also discuss how next-to-leading-order (NLO) corrections involving both s-wave and p-wave ${ }^{10}$ Be-neutron interactions affect our results, and display the NLO predictions for quantities which are free of additional short-distance operators at this order. Information on neutron- ${ }^{10}$ Be scattering in the relevant channels is inferred.
\end{abstract}

*Electronic address: hammer@hiskp.uni-bonn.de

$\dagger$ Electronic address: phillips@phy.ohiou.edu 


\section{INTRODUCTION}

The first excitation of the Beryllium-10 nucleus is $3.4 \mathrm{MeV}$ above the ground state, and that ground state has spin and parity quantum numbers $J^{P}=0^{+}$. Meanwhile, the Beryllium-11 nucleus has a $1 / 2^{+}$state whose neutron separation energy is $500 \mathrm{keV}$, and a $1 / 2^{-}$state whose neutron separation energy is $180 \mathrm{keV}$ [1]. The shallowness of these two states of ${ }^{11} \mathrm{Be}$ compared to the bound states of ${ }^{10} \mathrm{Be}$ suggests that they have significant components in which a loosely-bound neutron orbits a ${ }^{10}$ Be core. In this "one-neutron halo" picture the $1 / 2^{+}$is predominantly an s-wave bound state, while the $1 / 2^{-}$is predominantly a relative p-wave between the neutron and the core. In this paper, we discuss efforts to use effective field theory (EFT) to systematically implement such a halo picture of the ${ }^{11} \mathrm{Be}$ nucleus.

This halo viewpoint is reinforced by the fact that the scattering volume of $\mathrm{n}^{-10} \mathrm{Be}$ scattering in the $l=1, J=1 / 2$ channel has been determined to be [2]

$$
a_{1}=(457 \pm 67) \mathrm{fm}^{3} .
$$

The corresponding length scale of order $8 \mathrm{fm}$ is large compared to the natural length-scale of core-neutron interactions, which is $\approx 2-3 \mathrm{fm}$.

The datum (1), together with the information on the bound-state energies in the ${ }^{10} \mathrm{Be}$ and ${ }^{11}$ Be systems, helps us to estimate the expansion parameter in our Halo EFT. This is the binding energy of the halo nucleus, as compared to the energy required to excite the core, i.e. $B_{\mathrm{lo}} / B_{\mathrm{hi}} \approx 1 / 6$. Converting this to an estimate of the different distance scales involved, we infer that a majority of the probability density of ${ }^{11} \mathrm{Be}$ occupies a region outside the ${ }^{10} \mathrm{Be}$ core: $R_{\text {core }} / R_{\text {halo }} \approx 0.4$, which is consistent with the ratio implied by the numbers in the previous paragraph. This ratio of distance scales is the formal expansion parameter for the EFT, and since it is not particularly small, leading-order calculations are only a first step. We therefore present calculations up to next-to-leading order for several quantities, in order to confirm that the series is converging as expected.

In particular we apply this EFT to electromagnetic reactions in the ${ }^{11} \mathrm{Be}$ system. The $\mathrm{B}(\mathrm{E} 1)\left(1 / 2^{+} \rightarrow 1 / 2^{-}\right)$transition has recently been measured to be

$$
\mathrm{B}(\mathrm{E} 1)\left(1 / 2^{+} \rightarrow 1 / 2^{-}\right)=0.105(12) e^{2} \mathrm{fm}^{2}
$$

using intermediate-energy Coulomb excitation [3]. This is consistent with the older number

$$
\mathrm{B}(\mathrm{E} 1)\left(1 / 2^{+} \rightarrow 1 / 2^{-}\right)=0.116(12) e^{2} \mathrm{fm}^{2}
$$

from lifetime measurements [4]. There are also two recent data sets on the Coulomb-induced breakup of the ${ }^{11} \mathrm{Be}$ nucleus [5, 6] (see also Ref. [7]). Both experiments extracted the excitation function $d \mathrm{~B}(\mathrm{E} 1) / d E$ as a function of the energy of the outgoing neutron $E$. For low neutron energies this excitation function is affected by the final-state interaction in the p-waves, and can be predicted in the halo picture [2]. Ref. [5] also extracted a neutron radius for the ground state of ${ }^{11} \mathrm{Be}$ from their data:

$$
\left\langle r^{2}\right\rangle^{1 / 2}=5.7(4) \mathrm{fm}
$$

This is consistent with the recent atomic-physics measurement of the ${ }^{11}$ Be charge radius [8]:

$$
\left\langle r_{E}^{2}\right\rangle_{11 \mathrm{Be}}^{1 / 2}=2.463(16) \mathrm{fm} .
$$


All of these measurements can be addressed within the Halo EFT we will use here. In this theory the s- and p-wave states of the Beryllium-11 nucleus are generated by core-neutron contact interactions. The theory does not get the interior part of the nuclear wave function correct, but, by construction, it reproduces the correct asymptotics of the wave functions of these states:

$$
\begin{aligned}
& u_{0}(r)=A_{0} \exp \left(-\gamma_{0} r\right), \\
& u_{1}(r)=A_{1} \exp \left(-\gamma_{1} r\right)\left(1+\frac{1}{\gamma_{1} r}\right),
\end{aligned}
$$

for the $500 \mathrm{keV}$ and $180 \mathrm{keV}$ states, respectively. The quantities $\gamma_{0}$ and $\gamma_{1}$ are determined by the neutron separation energies of the states in question. At leading order (LO) in the expansion the Asymptotic Normalization Coefficients (ANCs) $A_{0}$ and $A_{1}$ are fixed. (In the case of the p-wave this is related to the theorem discussed in Ref. [9].) However, at nextto-leading order $A_{0}$ and $A_{1}$ become, in essence, free parameters of the theory, and must themselves be extracted from data.

Halo EFT is well-suited for this task. It is not intended to compete with ab initio calculations of this halo nucleus (see, e.g. [10, 11]) or of ${ }^{10}$ Be-n scattering [12], or with microscopic descriptions of the ${ }^{11} \mathrm{Be}$ E1 strength (see, e.g. 4]). Instead, Halo EFT is complementary to such approaches, since it takes $A_{0}, A_{1}, \gamma_{0}$, and $\gamma_{1}$ as input, rather than seeking to predict them via a detailed description of the $A=11$ system. The EFT's goal is to ensure that the long-distance properties of the halo are correctly taken care of, and then to elucidate the relationships between different observables in the ${ }^{10}$ Be-n system that result. Such correlations flow directly from the existence of the shallow $1 / 2^{+}$and $1 / 2^{-}$bound states in this system. In particular, below we will show how $A_{0}$ and $A_{1}$ are correlated with neutron- ${ }^{10}$ Be scattering observables, as well as with $\mathrm{B}(\mathrm{E} 1)\left(1 / 2^{+} \rightarrow 1 / 2^{-}\right)$and the Coulomb dissociation data. This, in turn, demonstrates how - and with what accuracy - the ANCs can be inferred from these experimental quantities.

A preliminary version of these findings appeared in Ref. [13]. The presentation here corrects and expands upon that earlier work. A related study of radiative neutron capture on Lithium-7 was recently carried out in [14]. The mechanism for the cancellation of divergences in the s-to-p transition in this reaction and in the Beryllium-11 system is the same.

\section{HALO EFT FOR BERYLLIUM-11}

We use the "Halo EFT" developed in Refs. [15, 16] to calculate the properties of the Beryllium-11 nucleus. The degrees of freedom in our Halo EFT treatment are the ${ }^{10}$ Be core and the neutron. The EFT expansion in this case is an expansion in powers of $\omega / B_{\text {high }}$. Here $B_{\text {high }}$ is, e.g. the excitation energy of states in ${ }^{10} \mathrm{Be}$, and so is of order a few $\mathrm{MeV}$, and $\omega$ is the energy of the photon exciting the electromagnetic transition of interest.

\section{A. Lagrangian: strong sector}

In our calculation, the ${ }^{10} \mathrm{Be}$ core and the neutron are represented by the bosonic field $c$ and a spinor field $n$, respectively. We include the strong s-wave and p-wave interactions that lead to the shallow bound states in the ${ }^{11}$ Be system through the incorporation of additional 
fields, which encode physics of these states. Therefore the $1 / 2^{+}$state is constructed as a spinor field, $\sigma_{s}$. In contrast, the field representing the $1 / 2^{-}$state is a pseudo-spinor, i.e. it is parity odd. We denote it as $\pi_{s}$. Its behavior under parity restricts the types of couplings which can appear in the Lagrangian. In particular, only combinations of nucleon and core fields with an odd number of derivatives can couple to $\pi$. Moreover, when constructing these operators we must decompose them into their irreducible representations under the rotation group. The portion that couples to $\pi_{s}$ is then just the $J=1 / 2$ part. For example, if we construct $n_{\beta}\left(i \stackrel{\leftrightarrow}{\nabla}_{j}\right) c$ where $\stackrel{\leftrightarrow}{\nabla}_{j}=(\overleftarrow{\nabla}-\vec{\nabla})_{j}$, this combination has both $J=1 / 2$ and $J=3 / 2$ parts. We project out the $J=1 / 2$ part by defining:

$$
[n(i \stackrel{\leftrightarrow}{\nabla}) c]_{\frac{1}{2}, s}=\sum_{\beta j}\left(\frac{1}{2} \beta 1 j \mid\left(\frac{1}{2} 1\right) \frac{1}{2} s\right) n_{\beta}(i \stackrel{\leftrightarrow}{\nabla} j) c,
$$

where $\left(j_{1} m_{1} j_{2} m_{2} \mid\left(j_{1} j_{2}\right) J M\right)$ is the Clebsch-Gordan coefficient to couple $j_{1}$ and $j_{2}$ to $J$.

With the masses of the neutron and the core are denoted by $m$ and $M$, respectively, and $M_{n c}=(M+m)$ is the total mass of the $\mathrm{n}-{ }^{10}$ Be system, we then have:

$$
\begin{aligned}
\mathcal{L}= & c^{\dagger}\left(i \partial_{t}+\frac{\nabla^{2}}{2 M}\right) c+n^{\dagger}\left(i \partial_{t}+\frac{\nabla^{2}}{2 m}\right) n \\
& +\sigma_{s}^{\dagger}\left[\eta_{0}\left(i \partial_{t}+\frac{\nabla^{2}}{2 M_{n c}}\right)+\Delta_{0}\right] \sigma_{s}+\pi_{s}^{\dagger}\left[\eta_{1}\left(i \partial_{t}+\frac{\nabla^{2}}{2 M_{n c}}\right)+\Delta_{1}\right] \pi_{s} \\
& -g_{0}\left[c^{\dagger} n_{s}^{\dagger} \sigma_{s}+\sigma_{s}^{\dagger} n_{s} c\right]-\frac{g_{1}}{2}\left(\pi_{s}^{\dagger}[n(i \stackrel{\leftrightarrow}{\nabla}) c]_{\frac{1}{2}, s}+\left[c^{\dagger}(i \stackrel{\leftrightarrow}{\nabla}) n^{\dagger}\right]_{\frac{1}{2}, s} \pi_{s}\right) \\
& -\frac{g_{1}}{2} \frac{M-m}{M_{n c}}\left(\pi_{s}^{\dagger}[i \vec{\nabla}(n c)]_{\frac{1}{2}, s}-\left[i \vec{\nabla}\left(n^{\dagger} c^{\dagger}\right)\right]_{\frac{1}{2}, s} \pi_{s}\right)+\ldots,
\end{aligned}
$$

where we adopt the convention that repeated spin indices are summed. Note that the last line of Eq. (8) represents an additional p-wave interaction necessary to maintain Galilean invariance. It is required because the $n$ and $c$ fields have different masses. The dots represent higher-order interactions not considered here. One such interaction involves the $J=3 / 2$ part of the $[n(i \stackrel{\leftrightarrow}{\nabla}) c]$ operator:

$$
\begin{aligned}
\mathcal{L}_{3 / 2} & =-\frac{C^{(3 / 2)}}{4}[n(i \stackrel{\leftrightarrow}{\nabla}) c]_{\frac{3}{2}, \beta}^{\dagger}[n(i \stackrel{\leftrightarrow}{\nabla}) c]_{\frac{3}{2}, \beta} \\
& =-\frac{C^{(3 / 2)}}{4} \sum_{\alpha s_{1} j s_{2} k}\left(\frac{1}{2} s_{1} 1 j \mid\left(\frac{1}{2} 1\right) \frac{3}{2} \alpha\right)\left(\frac{1}{2} s_{2} 1 k \mid\left(\frac{1}{2} 1\right) \frac{3}{2} \alpha\right)\left(c^{\dagger}\left(i \stackrel{\leftrightarrow}{\nabla}{ }_{j}\right) n_{s_{1}}^{\dagger}\right)\left(n_{s_{2}}(i \stackrel{\leftrightarrow}{\nabla} k) c\right) .
\end{aligned}
$$

As we shall discuss in the next section, this interaction is assumed to be natural in our power counting, in contrast to the interactions mediated by $\pi$ and $\sigma$ fields, which are enhanced.

\section{B. S-wave ${ }^{10}$ Be-neutron interactions}

In order to treat the shallow s-wave state in the ${ }^{10}$ Be-neutron system we adopt the counting that has been successfully developed to treat shallow s-wave states in the nucleonnucleon system [17 21]. In leading order, the $\sigma$ field is static and its bare propagator is simply 


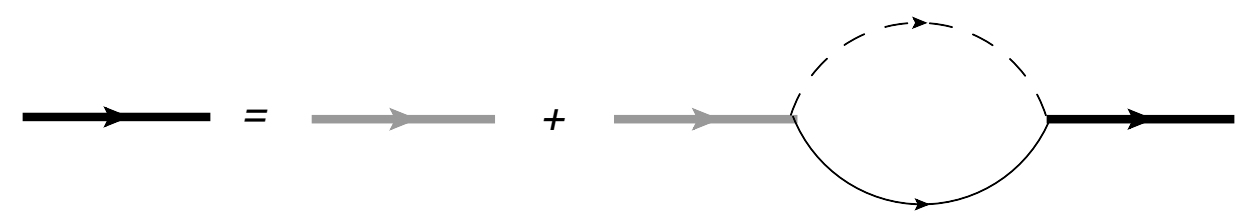

FIG. 1: Diagrammatic representation of the Dyson equation for the dressed $\sigma$ propagator representing the s-wave ${ }^{10} \mathrm{Be}-\mathrm{n}$ bound state in the theory. Here and below the dashed line indicates the field for the ${ }^{10} \mathrm{Be}$ core, and the thin solid line is the neutron. The thick grey line is the bare $\sigma$ propagator, and the thick black line is the dressed $\sigma$ propagator.

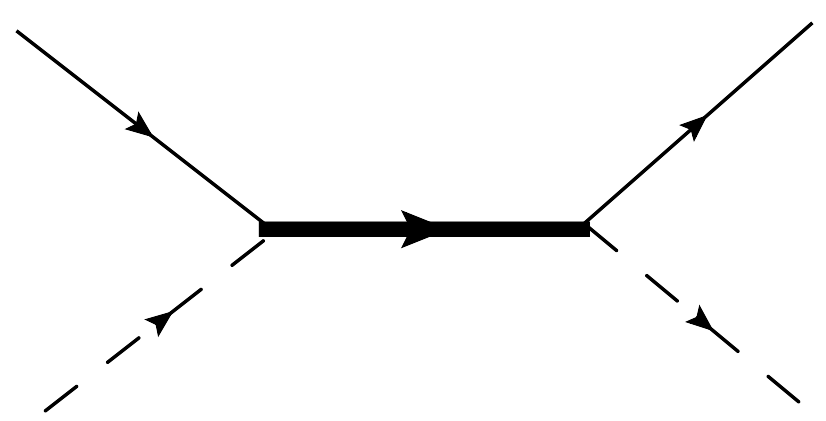

FIG. 2: The Feynman diagram relating the dressed $\sigma$ propagator to the s-wave neutron-core scattering amplitude.

$1 / \Delta_{0}$. Dividing out the leading term, the one-loop correction to the bare propagator is $g_{0}^{2} / \Delta_{0}$ times an $n c$ bubble which has a typical size of order $1 / R_{\text {halo }}{ }^{1}$. Since $g_{0}^{2} / \Delta_{0} \sim R_{\text {halo }}$, this correction is of order one. Consequently, the $n c$ loops must be resummed when computing the full $\sigma$ propagator.

This can be achieved through the Dyson equation shown in Fig. 1, which leads to:

$$
D_{\sigma}(p)=\frac{1}{\Delta_{0}+\eta_{0}\left[p_{0}-\mathbf{p}^{2} /\left(2 M_{n c}\right)+i \epsilon\right]-\Sigma_{\sigma}(p)},
$$

with $\Sigma_{\sigma}(p)$ the one-loop self-energy for the $\sigma$ field.

This one-loop self-energy is calculated as:

$$
\Sigma_{\sigma}(p)=-\frac{g_{0}^{2} m_{R}}{2 \pi}\left[i \sqrt{2 m_{R}\left(p_{0}-\frac{\mathbf{p}^{2}}{2 M_{n c}}\right)}+\mu\right],
$$

when computed in power-law divergence subtraction (PDS) with a scale $\mu$ [18, 19]. Here we have introduced the reduced mass of the neutron-core system:

$$
m_{R}=\frac{m M}{m+M}
$$

and the limit $\epsilon \rightarrow 0^{+}$in the end is understood.

\footnotetext{
${ }^{1}$ In a suitable regularization scheme, e.g. power-law divergence subtraction [18, 19], this is true for both the real and imaginary parts of the loops.
} 
Substituting Eq. (11) into Eq. (10), we can set the parameters $g_{0}$ and $\Delta_{0}$ by computing the s-wave neutron-core scattering amplitude in the theory defined by Eq. (8) (see Fig. 2):

$$
t_{0}(E)=g_{0}^{2} D_{\sigma}(E, \mathbf{0}),
$$

in the two-body center-of-mass frame with $E=k^{2} /\left(2 m_{R}\right)$. This is then matched to the effective-range expansion in this channel:

$$
t_{0}(E)=\frac{2 \pi}{m_{R}} \frac{1}{1 / a_{0}-\frac{1}{2} r_{0} k^{2}+i k},
$$

producing

$$
D_{\sigma}(p)=\frac{2 \pi \gamma_{0}}{m_{R}^{2} g_{0}^{2}} \frac{1}{1-r_{0} \gamma_{0}} \frac{1}{p_{0}-\frac{\mathbf{p}^{2}}{2 M_{n c}}+B_{0}}+R_{\sigma}(p),
$$

where $R_{\sigma}(p)$ is regular at the pole $p_{0}-\frac{\mathbf{p}^{2}}{2 M_{n c}}=-B_{0}$. In Eq. 15 , the position of the pole is determined by the binding energy $B_{0}=\gamma_{0}^{2} /\left(2 m_{R}\right)$, and $\gamma_{0}$ is the positive root of the equation:

$$
\frac{1}{a_{0}}+\frac{1}{2} r_{0} \gamma_{0}^{2}-\gamma_{0}=0
$$

The wave-function renormalization for the dressed $\sigma$ propagator can be read off as the residue of the pole in Eq. (15):

$$
Z_{\sigma}=\frac{2 \pi \gamma_{0}}{m_{R}^{2} g_{0}^{2}}\left(1-\gamma_{0} r_{0}\right)^{-1}
$$

This result is valid to NLO in the expansion in $R_{\text {core }} / R_{\text {halo }} \sim r_{0} \gamma_{0}$. It yields a wave function (6) with

$$
A_{0}=\sqrt{\frac{2 \gamma_{0}}{1-\gamma_{0} r_{0}}}
$$

\section{C. p-wave ${ }^{10}$ Be-neutron interactions}

We proceed similarly for the p-wave state. The propagator, $D_{\pi}(p)_{s s^{\prime}}=D_{\pi}(p) \delta_{s s^{\prime}}$, for this state obeys the Dyson equation depicted in Fig. 3. Rather than computing the self-energy

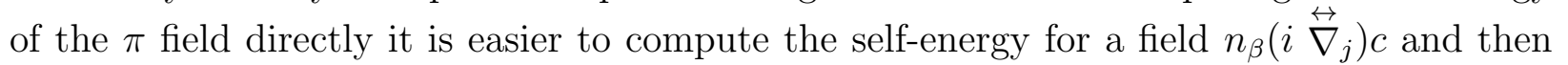
couple the neutron spin and the relative momentum in the appropriate way to project out the $J=1 / 2$ piece of the result. Hence we now consider the one-loop self-energy, $\Sigma_{\pi}(p)_{i j, \beta \alpha}$ for such a p-wave field. We first observe:

$$
\Sigma_{\pi}(p)_{i j, \alpha \beta}=\delta_{i j} \delta_{\alpha \beta} \Sigma(p) .
$$

The scalar function:

$$
\Sigma(p)=-\frac{m_{R} g_{1}^{2}}{6 \pi} 2 m_{R}\left(p_{0}-\frac{\mathbf{p}^{2}}{2 M_{n c}}\right)\left[i \sqrt{2 m_{R}\left(p_{0}-\frac{\mathbf{p}^{2}}{2 M_{n c}}+i \epsilon\right)}+\mu\right]
$$

where the PDS scheme has been employed and momentum traces have been performed in three dimensions. From this we can construct a self-energy for transitions from the $\pi$-field state $s$ to the $\pi$-field state $s^{\prime}$ :

$$
\Sigma_{\pi s^{\prime} s}(p)=\sum_{\beta j}\left(\frac{1}{2} \beta 1 j \mid\left(\frac{1}{2} 1\right) \frac{1}{2} s\right)\left(\frac{1}{2} \beta 1 j \mid\left(\frac{1}{2} 1\right) \frac{1}{2} s^{\prime}\right) \Sigma(p)
$$




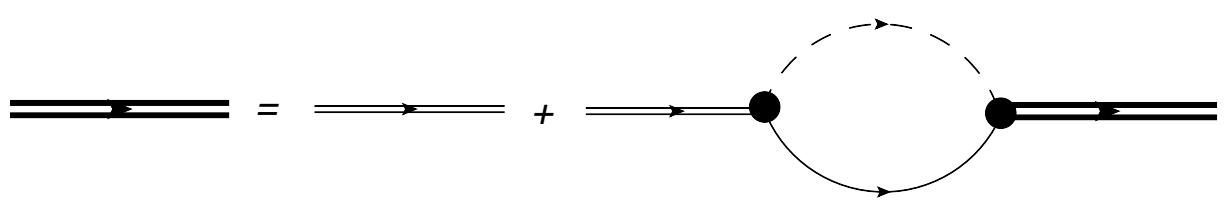

FIG. 3: Diagrammatic representation of the Dyson equation for the dressed $\pi$ propagator representing the p-wave ${ }^{10} \mathrm{Be}-\mathrm{n}$ bound state in the theory. Once again, the dashed line indicates the field for the ${ }^{10} \mathrm{Be}$ core, and the thin solid line is the neutron. The thin double line is the bare $\pi$ propagator, and the thick double line is the dressed $\pi$ propagator.

since $\Sigma$ is independent of $\beta$ and $j$ we can use completeness of the Clebsch-Gordon coefficients to show that $\Sigma$ is diagonal in $s^{\prime}$ and $s$, i.e. $\Sigma_{\pi s^{\prime} s}(p)=\delta_{s^{\prime} s} \Sigma(p)$. It follows that $D_{\pi}(p)$ takes the form:

$$
D_{\pi}(p)=\frac{1}{\Delta_{1}+\eta_{1}\left[p_{0}-\mathbf{p}^{2} /\left(2 M_{n c}\right)\right]-\Sigma(p)} .
$$

We note that since the self-energy loop is cubically divergent both parameters, $\Delta_{1}$ and $g_{1}$ are mandatory for renormalization at LO [15]. This time we are interested in the p-wave core-neutron scattering amplitude in the center-of mass frame:

$$
\begin{aligned}
t_{1}\left(\mathbf{p}^{\prime}, \mathbf{p} ; E\right) & =g_{1}^{2} \mathbf{p}^{\prime} \cdot \mathbf{p} D_{\pi}(E, \mathbf{0}) \\
& =\frac{6 \pi}{m_{R}} \frac{\mathbf{p}^{\prime} \cdot \mathbf{p}}{1 / a_{1}-\frac{1}{2} r_{1} k^{2}+i k^{3}},
\end{aligned}
$$

with $k=\sqrt{2 m_{R} E}=\left|\mathbf{p}^{\prime}\right|=|\mathbf{p}|$ for on-shell scattering. Consequently we obtain:

$$
D_{\pi}(p)=-\frac{6 \pi}{m_{R}^{2} g_{1}^{2}} \frac{1}{r_{1}+3 \gamma_{1}} \frac{1}{p_{0}-\mathbf{p}^{2} /\left(2 M_{n c}\right)+B_{1}}+\text { regular } .
$$

Here $\gamma_{1}=\sqrt{2 m_{R} B_{1}}$ is the solution of

$$
\frac{1}{a_{1}}+\frac{1}{2} r_{1} \gamma_{1}^{2}+\gamma_{1}^{3}=0
$$

where $a_{1}$ is the scattering volume, and $r_{1}$ the p-wave "effective range", which, in fact, has dimensions of $1 /$ length. Both parameters are required to leading order in the Halo EFT. The wave-function renormalization for the dressed $\pi$ propagator can be read off as the residue of the pole in Eq. (24):

$$
Z_{\pi}=-\frac{6 \pi}{m_{R}^{2} g_{1}^{2}} \frac{1}{r_{1}+3 \gamma_{1}} .
$$

The propagator (23) has three poles corresponding to the zeroes of Eq. (25). Using the NLO parameter values for the ${ }^{10}$ Be-n system obtained in Sec. II F below, we find two boundstate poles corresponding to typical momenta $\gamma_{1} \sim 1 / R_{\text {halo }}$ and $\gamma_{1} \sim 1 / R_{\text {core. }}$. The first is that which we identified with the ${ }^{11}$ Be excited state in the previous paragraph. The second is a spurious bound state, which is outside the domain of validity of Halo EFT, and is not physically realized in the ${ }^{11} \mathrm{Be}$ system. The third solution of Eq. 25 represents a virtual state with a typical momentum $\sim 1 / R_{\text {halo }}$.

The power counting for the propagator $D_{\pi}(p)$ that we adopt here is that of Ref. [16]. We take $r_{1} \sim 1 / R_{\text {core. }}$. The propagator then has a pole at $\gamma_{1} \sim 1 / R_{\text {halo, }}$, which occurs 
"kinematically" when $r_{1} k^{2} \sim 1 / a_{1}$. $a_{1}$ then must obviously be of order $R_{\text {halo }}^{2} R_{\text {core }}$ for such a "kinematic" pole to occur. We note that for $k \sim 1 / R_{\text {halo }}$ the unitarity piece of the propagator has a size $1 / R_{\text {halo }}^{3}$. Thus, away from the pole, the dominant contribution to the $\pi$ propagator now comes from the bare part (after appropriate renormalization) and so:

$$
t_{1}\left(\mathbf{p}^{\prime}, \mathbf{p} ; E\right)=\frac{6 \pi}{m_{R}} \frac{\mathbf{p}^{\prime} \cdot \mathbf{p}}{-\frac{1}{2} r_{1}\left(k^{2}+\gamma_{1}^{2}\right)},
$$

where we have also used Eq. 25 to re-express $1 / a_{1}$ in terms of $\gamma_{1}$ and then dropped the $\gamma_{1}^{3}$ term relative to the (larger) $r_{1} \gamma_{1}^{2}$ piece.

The result (27) can be easily re-expressed as:

$$
t_{1}\left(\mathbf{p}^{\prime}, \mathbf{p} ; E\right)=-\frac{6 \pi}{m_{R}^{2} r_{1}} \frac{\mathbf{p}^{\prime} \cdot \mathbf{p}}{E+B_{1}} .
$$

The amplitude (28) has only a pole at $E=-B_{1} \equiv-\gamma_{1}^{2} /\left(2 m_{R}\right)$-a pole that corresponds to the $1 / 2^{-}$state of ${ }^{11} \mathrm{Be}$. (This pole actually occurs on both sheets of the complex $E$ plane, since (27) exhibits poles in both the lower and upper half of the complex $k$-plane.) In contrast, the spurious deep pole from Eq. (23) has disappeared from the expressions (27) and (28). Our power counting therefore reproduces the spectrum of the ${ }^{11} \mathrm{Be}$ system. The p-state wave-function (6) is then obtained, with

$$
A_{1}=\sqrt{\frac{2 \gamma_{1}^{2}}{-r_{1}}}
$$

The p-wave phase shifts in this theory are given by:

$$
k^{3} \cot \delta_{1}=\gamma_{1}^{3}+\frac{1}{2} r_{1}\left(k^{2}+\gamma_{1}^{2}\right)
$$

if no expansions are made. However, for $r_{1} \sim 1 / R_{\text {core }}$ we can, once again, drop $\gamma_{1}^{3}$ to leading order in a power counting in $R_{\text {core }} / R_{\text {halo }}$, with the result that

$$
\cot \delta_{1}=\frac{r_{1}}{2}\left(\frac{1}{k}+\frac{\gamma_{1}^{2}}{k^{3}}\right)+O\left(\frac{R_{\text {core }}}{R_{\text {halo }}}\right) .
$$

Since $r_{1} \gg k, \gamma_{1}$ we have cot $\delta_{1}$ large, which implies that $\delta_{1}$ is approximately zero. Indeed

$$
\delta_{1}=\frac{2}{r_{1}} \frac{k^{3}}{k^{2}+\gamma_{1}^{2}}+O\left(\frac{R_{\text {core }}}{R_{\text {halo }}}\right),
$$

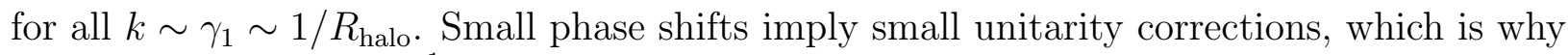
the imaginary part of $t_{1}^{-1}$ can be treated perturbatively in this regime.

The only exception to this occurs if we consider $\left|E-B_{1}\right| \sim \frac{\gamma_{1} B_{1}}{r_{1}}$. In that case we are close to the pole and the two terms $\sim\left(R_{\text {core }} R_{\text {halo }}^{2}\right)^{-1}$ in Eq. (27) cancel, or come close to doing so. It then becomes necessary to resum the pieces $\sim k^{3}$ and $\sim \gamma_{1}^{3}$ which were dropped in order to obtain Eq. (28) [16, 22]. In particular, if $B_{1}<0$ (i.e. the pole is at positive energy) then these corrections shift the pole off the real axis and mean that it represents a resonance. The propagator 23 thus describes a p-wave resonance if the pole is at positive energy and the width of the resonance at energy $E_{R}$ is of order $k_{R} / r_{1} E_{R}$ [15, 16, 23]. This is thus a narrow resonance if $r_{1} \sim 1 / R_{\text {core }}$. The case of p-wave resonances will not be discussed further here, since we will restrict ourselves to $B_{1}>0$, as is relevant for ${ }^{11} \mathrm{Be}$. This is the case of a shallow p-wave bound state. 


\section{Rescaled fields and naive dimensional analysis}

At this point it is useful to rescale the piece of the Lagrangian which encodes the enhanced s- and p-wave interactions. We rewrite $\mathcal{L}$ in terms of fields with non-canonical dimensions, which absorb factors of $g_{0}, g_{1}, m$, and $M[24]$. We define:

$$
\tilde{\sigma}_{s}=\sigma_{s} g_{0} m_{R} ; \quad \tilde{\pi}_{s}=\pi_{s} g_{1} m_{R} .
$$

The scaling of the absorbed factors $g_{0} m_{R}$ and $g_{1} m_{R}$ can be obtained by recalling the matching between (13) and (14) for s-waves, and in Eq. (23) for p-waves. At leading order, this yields:

$$
g_{0}^{2} m_{R}^{2} \simeq-\frac{2 \pi \eta_{0}}{r_{0}}, \quad g_{1}^{2} m_{R}^{2} \simeq-\frac{6 \pi \eta_{1}}{r_{1}}
$$

In our counting, we have $r_{0} \sim 1 / r_{1} \sim R_{\text {core }}$, which then determines how $g_{0}$ and $g_{1}$ scale with

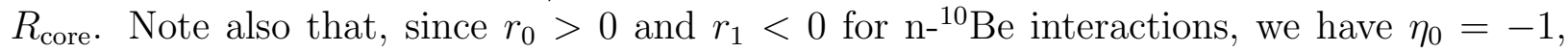
$\eta_{1}=1$ in this system.

We analyze $M_{n c} \mathcal{L}$ because this operator is dimension 5 , and has no powers of the mass in it anymore [25]. The expression for the product of the total mass and the pertinent piece of the Lagrangian then becomes, in terms of these fields:

$$
\begin{aligned}
M_{n c} \mathcal{L}= & \frac{1}{g_{0}^{2} m_{R}^{2}} \tilde{\sigma}_{s}^{\dagger}\left[\eta_{0}\left(i M_{n c} \partial_{t}+\nabla^{2}\right)+M_{n c} \Delta_{0}\right] \tilde{\sigma}_{s}-\frac{M_{n c}}{m_{R}}\left[c^{\dagger} n_{s}^{\dagger} \tilde{\sigma}_{s}+\tilde{\sigma}_{s}^{\dagger} n_{s} c\right] \\
& +\frac{1}{g_{1}^{2} m_{R}^{2}} \tilde{\pi}_{s}^{\dagger}\left[\eta_{1}\left(i M_{n c} \partial_{t}+\nabla^{2}\right)+M_{n c} \Delta_{1}\right] \tilde{\pi}_{s} \\
& -\frac{M_{n c}}{2 m_{R}}\left(\tilde{\pi}_{s}^{\dagger}[n(i \stackrel{\leftrightarrow}{\nabla}) c]_{\frac{1}{2}, s}+\left[c^{\dagger}(i \stackrel{\leftrightarrow}{\nabla}) n^{\dagger}\right]_{\frac{1}{2}, s} \tilde{\pi}_{s}\right)
\end{aligned}
$$

where the pieces of $\mathcal{L}$ that restore Galilean invariance have been suppressed. In terms of these new fields all the coefficients - even those in the "enhanced" interactions which generate shallow bound states (and resonances) are natural. The shallowness of these states is now encoded in the fact that the fields associated with them have non-canonical dimensions: $[\tilde{\sigma}]=2,[\tilde{\pi}]=1$, and non-canonical wave-function normalization-even at tree level.

\section{E. Lagrangian: electromagnetic sector}

Photons are then included in the Lagrangian via minimal substitution:

$$
\partial_{\mu} \rightarrow D_{\mu}=\partial_{\mu}+i e \hat{Q} A_{\mu}
$$

The charge operator $\hat{Q}$ takes different values, depending on whether it is acting on a $c$ field or an $n$ field. $\hat{Q} n=0$ for the neutron, and below we denote the eigenvalue of the operator $\hat{Q}$ for the $c$ field as $\hat{Q} c=Q_{c} c$. $Q_{c}=4$ in the case of interest here, where the core is Beryllium-10.

Here our focus is on electric properties (and form factors), and the dominant pieces of the electric response can be derived by looking at how the Lagrangian (8) is affected by the substitution (36). But, at higher orders in the computation of these properties, operators involving the electric field $\mathbf{E}$ and the fields $c, n, \sigma$, and $\pi$ which are gauge invariant by 
themselves contribute to observables. Possible one- and two-derivative operators with one power of the photon field are:

$$
\begin{aligned}
\mathcal{L}_{E M}= & -L_{C 0}^{(\sigma)} \sigma_{l}^{\dagger}\left(\nabla^{2} A_{0}-\partial_{0}(\nabla \cdot \mathbf{A})\right) \sigma_{l}-L_{E 1}^{(1 / 2)} \sum_{l l^{\prime} j} \sigma_{l} \pi_{l^{\prime}}^{\dagger}\left(\frac{1}{2} l \frac{1}{2} l^{\prime} \mid 1 j\right)\left(\nabla_{j} A_{0}-\partial_{0} A_{j}\right) \\
& -L_{C 0}^{(\pi)} \pi_{l}^{\dagger}\left(\nabla^{2} A_{0}-\partial_{0}(\nabla \cdot \mathbf{A})\right) \pi_{l} \\
& -L_{E 1}^{(3 / 2)} \sum_{l l^{\prime} j} \sigma_{l}[n(i \stackrel{\leftrightarrow}{\nabla}) c]_{3 / 2 l^{\prime}}^{\dagger}\left(\frac{1}{2} l \frac{3}{2} l^{\prime} \mid 1 j\right)\left(\nabla_{j} A_{0}-\partial_{0} A_{j}\right) .
\end{aligned}
$$

Note that if magnetic properties are to be considered we would also include operators involving $\partial_{i} A_{j}-\partial_{j} A_{i}$ and the neutron, core, and bound-state fields.

The electric interactions in Eq. (37) are gauge invariant by themselves, and so we must determine the order at which they occur. To do this we rewrite the Lagrangian (37) in terms of the rescaled fields (33). In terms of these fields we assume scaling by naive dimensional analysis with respect to the scale $R_{\text {core }}$ of the operators that appear in $M_{n c} \mathcal{L}$. We then obtain the following scaling of the coefficients written above:

$$
\begin{aligned}
L_{C 0}^{(\sigma)} & \sim R_{\text {core }}^{3} l_{C 0}^{(\sigma)} g_{0}^{2} m_{R}^{2}, \\
L_{E 1}^{(1 / 2)} & \sim R_{\text {core }} l_{E 1}^{(1 / 2)} g_{0} g_{1} m_{R}^{2}, \\
L_{C 0}^{(\pi)} & \sim R_{\text {core }} l_{C 0}^{(\pi)} g_{1}^{2} m_{R}^{2}, \\
L_{E 1}^{(3 / 2)} & \sim R_{\text {core }}^{4} l_{E 1}^{(3 / 2)} g_{0} m_{R},
\end{aligned}
$$

where the parameters $l \ldots$ are all of order one.

Below we show that the leading effects in the $\mathrm{E} 1\left(1 / 2^{+} \rightarrow 1 / 2^{-}\right)$matrix element have parametric dependence $R_{\text {halo }} \sqrt{\frac{R_{\text {core }}}{R_{\text {halo }}}}$. Including the proper wave-function renormalization factors, the operator $\sim L_{E 1}^{(1 / 2)}$ yields an effect $\sim R_{\text {core }} \sqrt{\frac{R_{\text {core }}}{R_{\text {halo }}}}$, and so occurs already at NLO in that quantity. Similarly the leading effects in the charge-radius-squared of the $1 / 2^{-}$state in ${ }^{11}$ Be are $\sim R_{\text {halo }} / r_{1} \sim R_{\text {halo }} R_{\text {core }}$. The operator proportional to $L_{C 0}^{(\pi)}$ above produces effects in this charge radius of order $R_{\text {core }} / r_{1} \sim R_{\text {core }}^{2}$, and so affects the prediction for the p-wave radius at next-to-leading order. Thus if we desire NLO accuracy for quantities involving the shallow p-wave bound state there are two parameters in the Halo EFT description of the electric structure of the Beryllium-11 which cannot be fixed from ${ }^{10}$ Be-neutron scattering information alone.

\section{F. Fixing parameters}

Using the values $B_{0}=500 \mathrm{keV}, B_{1}=180 \mathrm{keV}$ from Ref. [1, we infer $\gamma_{0}=0.15 \mathrm{fm}^{-1}$, and $\gamma_{1}=0.09 \mathrm{fm}^{-1}$, which are both of the expected size $1 / R_{\text {halo }}$. From the power counting discussed in Sec. IIC, we have at leading order:

$$
r_{1}=-\frac{2}{\gamma_{1}^{2} a_{1}} .
$$

It follows that if we adopt the value extracted in Ref. [2] from experimental data, Eq. (1), we have $r_{1}=(-0.54 \pm 0.08) \mathrm{fm}^{-1}$. This number should, however, be taken as indicative 
rather than definitive, since in the end we will fit $r_{1}$ to data at both LO and NLO, and deduce corresponding values for $a_{1}$ from our results. But already we see that the order of magnitude estimate implied by our counting $r_{1} \sim 1 / R_{\text {core }}$ is borne out by the numbers.

At NLO, Eq. 42 is corrected to:

$$
r_{1}=-\frac{2}{\gamma_{1}^{2} a_{1}}-2 \gamma_{1}
$$

which, if we again use Eq. (1) to get an idea of the effect, alters $r_{1}$ to $(-0.72 \pm 0.08) \mathrm{fm}^{-1}$. Such a $\sim 30 \%$ correction is in line with the anticipated expansion parameter of Halo EFT in the ${ }^{11}$ Be system.

In the s-waves the situation is more straightforward: there we count $a_{0} \sim R_{\text {halo }}$, and

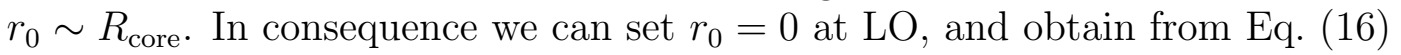

$$
\gamma_{0}=\frac{1}{a_{0}}
$$

At leading order, in s-waves we have:

$$
Z_{\sigma}=\frac{2 \pi \gamma_{0}}{m_{R}^{2} g_{0}^{2}}
$$

and all other pertinent results can be obtained by taking the $r_{0} \rightarrow 0$ limit of the formulae in Sec. IIB.

Thus, the parameters in Halo EFT for Beryllium-11 bound states at LO are $r_{1}, \gamma_{0}$ (or equivalently $a_{0}$ ), and $\gamma_{1}$. At NLO these are to be supplemented by $r_{0}$, and the electromagnetic contact interactions for the $1 / 2^{+} \rightarrow 1 / 2^{-}$E1 transition and $1 / 2^{-}$-state radius.

\section{RESULTS FOR BOUND-STATE OBSERVABLES}

Using Eq. (8) plus minimal substitution (36), we obtain a Lagrangian that describes interactions amongst the core, the neutron, the ground and excited states of the ${ }^{11}$ Be nucleus, and photons. In this section we use this Lagrangian to compute the form factor of the $1 / 2^{+}$ and $1 / 2^{-}$states and the E1 transition from the $1 / 2^{+}$to the $1 / 2^{-}$state.

\section{A. s-wave form factor}

The s-wave form factor is computed by calculating the contribution to the irreducible vertex for $A_{0} \sigma \sigma$ interactions shown in Fig. 4. This is the only diagram it is necessary to consider at leading order. After the application of wave-function renormalization, the irreducible vertex for the $A_{0}$ photon coupling to the $\sigma$ state is equal to $-i e Q_{c} G_{E}(|\mathbf{q}|)$, where $\mathbf{q}$ is the three-momentum of the virtual photon. (Such an interpretation is valid provided the computation is carried out in the Breit frame, where the four-momentum of the virtual photon $q=(0, \mathbf{q})$.) A straightforward calculation yields:

$$
G_{E}^{(\sigma)}(|\mathbf{q}|)=\frac{2 \gamma_{0}}{f|\mathbf{q}|} \arctan \left(\frac{f|\mathbf{q}|}{2 \gamma_{0}}\right)
$$




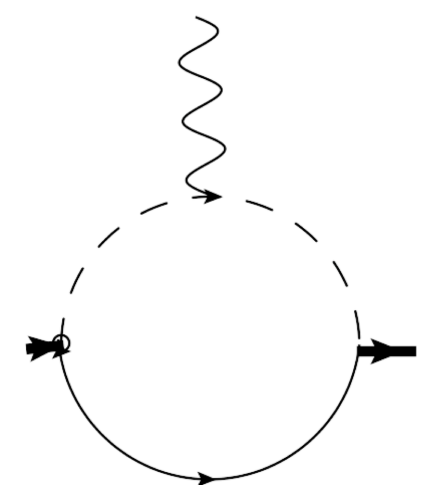

FIG. 4: The LO contribution to the irreducible vertex for an $A_{0}$ photon to couple to the field representing the ${ }^{10} \mathrm{Be}$-neutron s-wave bound state. Note that there is no diagram for the photon to couple to the neutron as this order, since $Q_{n}=0$.

with $f=m / M_{n c}=m_{R} / M$. Note that $G_{E}(0)=1$, as it should. For the deuteron, we have $f=1 / 2$, and this reduces to the LO result of Ref. [26].

The electric radius of the s-wave state can be extracted according to:

$$
G_{E}^{(\sigma)}(|\mathbf{q}|) \equiv 1-\frac{1}{6}\left\langle r_{E}^{2}\right\rangle^{(\sigma)} \mathbf{q}^{2}+\ldots
$$

and an expansion of Eq. (46) in powers of $|\mathbf{q}|$ then yields

$$
\left\langle r_{E}^{2}\right\rangle^{(\sigma)}=\frac{f^{2}}{2 \gamma_{0}^{2}}
$$

Eq. (48) gives the electric radius of the ${ }^{11} \mathrm{Be}$ ground state relative to the electric radius of ${ }^{10} \mathrm{Be}$. In order to compare with the experimental radius, we therefore have to add our result and the charge radius of ${ }^{10} \mathrm{Be}$ in quadrature:

$$
\left\langle r_{E}^{2}\right\rangle^{{ }_{11} \mathrm{Be}}=\left\langle r_{E}^{2}\right\rangle^{10} \mathrm{Be}+\frac{f^{2}}{2 \gamma_{0}^{2}} .
$$

This relation can be derived by writing the charge distribution of ${ }^{11} \mathrm{Be}$ as a convolution of the charge distribution of ${ }^{10} \mathrm{Be}$ with that of the ${ }^{10} \mathrm{Be}-\mathrm{n}$ halo system. Using the convolution theorem for the Fourier transform, one finds that the total rms radius squared can be written as the sum of the squared radii for ${ }^{10} \mathrm{Be}$ and for the ${ }^{10} \mathrm{Be}-\mathrm{n}$ halo system. The latter effect can be calculated in the Halo EFT. Inserting the value $\gamma_{0}=0.15 \mathrm{fm}^{-1}$ obtained in the previous section and using the experimental result for the ${ }^{10} \mathrm{Be}$ charge radius [8], we find $\left\langle r_{E}^{2}\right\rangle_{11 \mathrm{Be}}^{1 / 2}=2.40 \mathrm{fm}$ from this leading-order HEFT computation. This is $2-3 \%$ smaller than the atomic physics measurement which yields $\left\langle r_{E}^{2}\right\rangle_{11 \mathrm{Be}}^{1 / 2}=2.463(16)$ fm [8]. In fact, comparing our result for $\left\langle r_{c}^{2}\right\rangle^{11 \mathrm{Be}}-\left\langle r_{c}^{2}\right\rangle^{10} \mathrm{Be}\left(0.19 \mathrm{fm}^{2}\right)$ with the experimental result for this quantity $\left(0.51(17) \mathrm{fm}^{2}\right)$, the agreement looks poor. But, this difference is actually consistent with the nominal $40 \%$ size of NLO effects when Halo EFT is applied to this system.

At NLO a careful treatment of current conservation, which includes an operator associated with gauging the term $\sim \sigma^{\dagger} \partial_{0} \sigma$ in Eq. (8), still yields $G_{E}(0)=1$, but also produces an increased charge radius, as long as $r_{0}>0$, cf. Ref. [24, 27]:

$$
\left\langle r_{E}^{2}\right\rangle_{{ }^{11} \mathrm{Be}}=\left\langle r_{E}^{2}\right\rangle^{10 \mathrm{Be}}+\frac{f^{2}}{2\left(1-r_{0} \gamma_{0}\right) \gamma_{0}^{2}} .
$$


Therefore NLO corrections improve the agreement with experiment. The precise size of the increase is fixed once the s-wave effective range $r_{0}$ is determined, as we shall do in Sec. V below.

We can also obtain from our leading-order calculation a number for the mean-square of the relative core-neutron co-ordinate, $\left\langle r^{2}\right\rangle$, i.e.:

$$
\left\langle r^{2}\right\rangle=\frac{1}{2 \gamma_{0}^{2}}
$$

To convert this to a neutron radius, we must insert the conversion factor $m_{c} /\left(m_{c}+m_{n}\right)$. When this is done we find a $\mathrm{LO}$ neutron radius for the ${ }^{11} \mathrm{Be}$ ground state of:

$$
\left\langle r_{n}^{2}\right\rangle^{1 / 2}=4.3 \mathrm{fm}
$$

The neutron radius can be measured with a probe that couples only to the neutrons. To a very good approximation, the weak gauge boson $Z^{0}$ constitutes such a probe. Thus one could, in principle, measure the rms neutron radius using parity-violating electron scattering - c.f. the PREX experiment for the case of Lead-208 [28]. However, a measurement of parity-violating electron scattering from ${ }^{11} \mathrm{Be}$ is certainly beyond present-day experimental capabilities.

\section{B. p-wave form factor}

In this subsection, we calculate the charge form factor of the $1 / 2^{-}$excited state in ${ }^{11} \mathrm{Be}$. NLO corrections might be expected to be smaller there since its binding energy, and so its typical momentum, is lower. However, as we shall see, a counterterm enters already at NLO in this observable.

The p-wave form factor is computed by calculating the contribution to the irreducible vertex for $A_{0} \pi \pi$ interactions shown in Fig. 5. There are two diagrams at LO. The first diagram is analogous to that for the s-wave state while the second diagram represents a direct coupling of the photon to the $\pi$ field. The latter contribution is leading order for the $\mathrm{p}$-wave state since the effective range $r_{1}$ is leading order for this state.

As with the self-energy of the $\pi$-field, it is easier to compute the irreducible bubble for an $A_{0}$ photon coupling to the p-wave field $n_{\beta}\left(i \stackrel{\leftrightarrow}{\nabla}_{j}\right) c$. In this way, we find that, after the application of wave-function renormalization, the irreducible vertex for the $A_{0}$ photon coupling to the $1 / 2^{-}$state in the Breit frame can be written as:

$$
\begin{array}{r}
\left\langle\pi_{s}^{\prime}\left(\mathbf{p}^{\prime}\right)\left|J^{0}\right| \pi_{s}(\mathbf{p})\right\rangle=-i e Q_{c} \sum_{\alpha \beta i j}\left(\frac{1}{2} \alpha 1 i \mid\left(\frac{1}{2} 1\right) \frac{1}{2} s\right)\left(\frac{1}{2} \beta 1 j \mid\left(\frac{1}{2} 1\right) \frac{1}{2} s\right) \\
\delta_{\alpha \beta}\left[G_{E}^{(\pi)}(|\mathbf{q}|) \delta_{i j}+\frac{1}{2 M_{n c}^{2}} G_{Q}^{(\pi)}(|\mathbf{q}|)\left(q_{i} q_{j}-\frac{\mathbf{q}^{2} \delta_{i j}}{3}\right)\right],
\end{array}
$$

where $\mathbf{q}=\mathbf{p}^{\prime}-\mathbf{p}$ is the three-momentum of the virtual photon. Here we have expressed the form factor in terms of the charge and quadrupole form factors of a vector field. Choosing $\mathbf{q}=|\mathbf{q}| \hat{z}$, and exploiting the fact that the neutron spin is unaffected by the charge operators that can occur up to the order we consider here, a brief calculation shows

$$
\left\langle\pi_{s}^{\prime}\left(\mathbf{p}^{\prime}\right)\left|J^{0}\right| \pi_{s}(\mathbf{p})\right\rangle=-i e Q_{c} \delta_{s^{\prime} s} G_{E}^{(\pi)}(|\mathbf{q}|)
$$




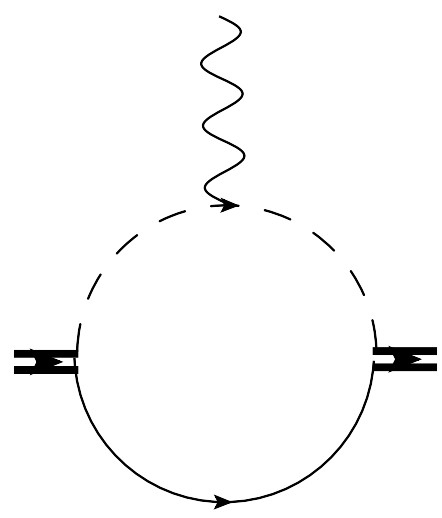

(a)

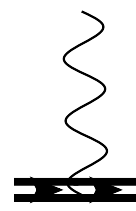

(b)

FIG. 5: The LO diagrams contributing to the irreducible vertex for an $A_{0}$ photon to couple to the $\pi$ field representing the ${ }^{10} \mathrm{Be}$-neutron p-wave bound state. Diagram (a) is analog to the s-wave form factor while diagram (b) arises since the effective range $r_{1}$ enters at leading order for the p-wave state.

The quadrupole form factor is thus unobservable in the $1 / 2^{-}$state. It could be observed in a $3 / 2^{-}$state. The lowest $3 / 2^{-}$state in ${ }^{11} \mathrm{Be}$ is $2.69 \mathrm{MeV}$ above the ground state. However, this state is a $\mathrm{n}^{10} \mathrm{Be}$ scattering resonance that corresponds to typical momenta of order $1 / R_{\text {core }}$ and thus is outside the range of applicability of the Halo EFT. Since the situation could be different in other one-neutron halo nuclei, we quote the result for the quadrupole form factor for completeness:

$$
G_{Q}^{(\pi)}(|\mathbf{q}|)=\frac{2 M_{n c}^{2}}{r_{1}+3 \gamma_{1}} \frac{3}{4|\mathbf{q}|^{3} f}\left(2|\mathbf{q}| f \gamma_{1}+\left(|\mathbf{q}|^{2} f^{2}-4 \gamma_{1}^{2}\right) \arctan \left(\frac{f|\mathbf{q}|}{2 \gamma_{1}}\right)\right)
$$

In the case of ${ }^{11} \mathrm{Be}$, only the charge form factor is observable. A straightforward calculation yields:

$$
G_{E}^{(\pi)}(|\mathbf{q}|)=\frac{1}{r_{1}+3 \gamma_{1}}\left[r_{1}+\frac{1}{|\mathbf{q}| f}\left(2|\mathbf{q}| f \gamma_{1}+\left(|\mathbf{q}|^{2} f^{2}+2 \gamma_{1}^{2}\right) \arctan \left(\frac{f|\mathbf{q}|}{2 \gamma_{1}}\right)\right)\right]
$$

where again $f=m / M_{n c}=m_{R} / M$. For a strict LO result $r_{1}+3 \gamma_{1}$ should be replaced by $r_{1}$ in these expressions.

We have $G_{E}^{(\pi)}(0)=1$, as required by charge conservation. The electric radius of the p-wave state relative to the ${ }^{10}$ Be ground state can be extracted according to Eq. (47), and we obtain the electric radius for the case $r_{1} \sim \gamma_{1}$ from an expansion of Eq. (56) in powers of $|\mathbf{q}|$ :

$$
\left\langle r_{E}^{2}\right\rangle^{(\pi)}=-\frac{5 f^{2}}{2 \gamma_{1}\left(3 \gamma_{1}+r_{1}\right)} .
$$

At LO in the situation of interest here $\left|r_{1}\right| \gg \gamma_{1}$, we should replace this by:

$$
\left\langle r_{E}^{2}\right\rangle^{(\pi)}=-\frac{5 f^{2}}{2 \gamma_{1} r_{1}}=\frac{5 f^{2} a_{1}^{\mathrm{LO}} \gamma_{1}}{4}
$$


This is, as promised above, $\sim R_{\text {halo }} R_{\text {core }}$.

It is interesting to examine why the p-wave radius is more sensitive to short-distance physics than the s-wave one. From the co-ordinate space point of view we find that at LO the radius of the p-wave state must be calculated as:

$$
\left\langle r_{E}^{2}\right\rangle^{(\pi)}=A_{1}^{2} f^{2} \int_{0}^{\infty} d r r^{2}\left(1+\frac{1}{\gamma_{1} r}\right)^{2} e^{-2 \gamma_{1} r} .
$$

Inserting $A_{1}$ from Eq. (29) yields Eq. (58). Since the integral is finite, we can compute the contribution to it from values of $r \ll 1 / \gamma_{1}$ :

$$
\left\langle r_{E}^{2}\right\rangle_{S D}^{(\pi)}=A_{1}^{2} f^{2} \int_{0}^{R_{\text {core }}} d r r^{2}\left(1+\frac{1}{\gamma_{1} r}\right)^{2} e^{-2 \gamma_{1} r},
$$

which produces, for $R_{\text {core }} \ll 1 / \gamma_{1}$, a short-distance fraction of the total result of:

$$
\frac{\left\langle r_{E}^{2}\right\rangle_{S D}^{(\pi)}}{\left\langle r_{E}^{2}\right\rangle^{(\pi)}} \sim R_{\text {core }} \gamma_{1}
$$

The parametric dependence of the short-distance contribution on $R_{\text {core }}$ is in accord with the result obtained in the previous section for the corresponding counterterm using naive dimensional analysis in our rescaled Lagrangian.

It might seem counterintuitive that there is a short-distance contribution to $\left\langle r_{E}^{2}\right\rangle^{(\pi)}$ already at NLO - especially when the corresponding effect does not occur in $\left\langle r_{E}^{2}\right\rangle^{(\sigma)}$ until N $\mathrm{N}^{3} \mathrm{LO}$ (see Eq. (38) and Ref. [26]). The physics of this is, however, quite straightforward. It is associated with the propensity of the p-wave state's probability distribution to be drawn in to shorter distances than the s-wave one, as it gets caught between the attractive potential that produces the excited state of ${ }^{11} \mathrm{Be}$ and the centrifugal barrier. Observables associated with a shallow p-wave bound state will, therefore, generically exhibit counterterms at lower order than those of their s-wave counterparts.

Numerical evaluation of the LO expression (58) leads to the prediction for the charge radius of the ${ }^{11} \mathrm{Be} \mathrm{p}$-wave state relative to the ${ }^{10} \mathrm{Be}$ ground state $\left\langle r_{c}^{2}\right\rangle^{(\pi)}=0.36 \mathrm{fm}^{2}$ at LO. (Here we have used as input the value $r_{1}=-0.66 \mathrm{fm}$, see the next section.) A trivial way to estimate the size of NLO corrections is to use the result for $A_{1}$ in the limit $\left|r_{1}\right| \sim \gamma_{1}$ :

$$
A_{1}=\sqrt{-\frac{2 \gamma_{1}^{2}}{r_{1}+3 \gamma_{1}}}
$$

instead of Eq. 29). This yields a $20 \%$ correction at NLO in agreement with the expectation from the power counting, although it must be remembered that the entire NLO result in the situation where $\left|r_{1}\right| \gg \gamma_{1}$ includes the contributions of the operator $\sim \pi_{s}^{\dagger} \nabla^{2} A_{0} \pi_{s}$. Thus the only prediction we can offer here is a leading-order one. Using again the experimental result for the ${ }^{10} \mathrm{Be}$ charge radius [8], we predict the electric radius of the $1 / 2^{-}$state as:

$$
\left\langle r_{E}^{2}\right\rangle_{11}^{1 / 2} \mathrm{Be}^{*}=(2.43 \pm 0.1) \mathrm{fm}
$$

where our error bar comes from the above estimate of NLO effects. To our knowledge there is, as yet, no experimental determination of the charge radius of this state. Note that 
in Eq. 63 we are assuming that the short-distance effects in $\left\langle r_{E}^{2}\right\rangle_{11}^{1 / 2} \mathrm{Be}^{*}$ scale with $f$, as suggested by the renormalization-group argument summarized in Eq. (60). Short-distance effects which do not involve a factor of $f$, e.g., modification of the proton distribution of ${ }^{10} \mathrm{Be}$ in the ${ }^{11} \mathrm{Be}$ excited state due to a non-recoil effect, could have an impact on our final result that is larger than $0.1 \mathrm{fm}$.

\section{E1 transition: $1 / 2^{+} \rightarrow 1 / 2^{-}$state}

Now we consider the E1 transition from the $1 / 2^{+}$state to the $1 / 2^{-}$state. The irreducible vertex for this transition is depicted in Fig. 6. We compute the transition for a photon of arbitrary four momentum $k=(\omega, \mathbf{k})$, and the sum of diagrams yields $-i \Gamma_{s^{\prime} s \mu}$ where $s^{\prime}(s)$ is the spin projection of the $1 / 2^{-}\left(1 / 2^{+}\right)$state and $\mu$ is the polarization index of the photon.

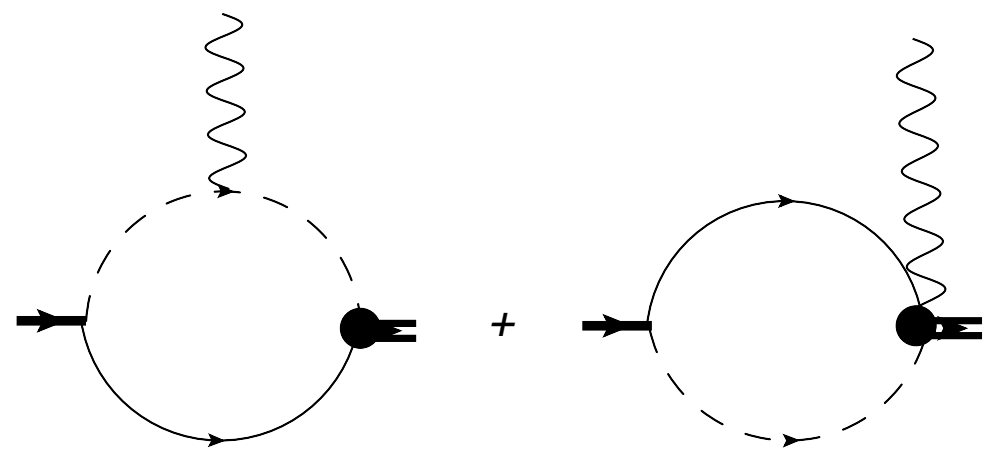

FIG. 6: The two diagrams needed for the irreducible vertex that governs the s-to-p-state transition, $\Gamma_{j \mu}$ in Halo EFT at leading order.

We first observe that both the diagrams depicted in Fig. 6 are divergent, but that the divergences cancel, as they should since gauge invariance precludes us from writing down any contact interaction that contributes to this observable at leading order. Current conservation at LO can be explicitly checked, and we find that, as long as both diagrams are considered [13]:

$$
k^{\mu} \Gamma_{s^{\prime} s \mu}=0 .
$$

Note that if only the long-distance E1 mechanism on the left-hand side of Fig. 6 is considered, as was done, for example, in Ref. [29], then current conservation is not satisfied, and it appears that some input from short-distance physics is needed in order to define the prediction for this observable.

Since we are considering electric properties, and the spin of the neutron is not affected by the photon interaction, it is, once again, convenient to re-express $\Gamma_{s^{\prime} s \mu}$ in terms of the vertex function for specific components of the p-wave interaction that appears at the $\pi$-neutron-core vertex:

$$
\Gamma_{s^{\prime} s \mu}=\sum_{j}\left(\frac{1}{2} s 1 j \mid\left(\frac{1}{2} 1\right) \frac{1}{2} s^{\prime}\right) \tilde{\Gamma}_{j \mu} .
$$

We note that if we examine the case $s^{\prime}=s$ then only $j=0$ contributes to the sum in Eq. 65), and we have

$$
-\Gamma_{++\mu}=\Gamma_{--\mu}=\frac{1}{\sqrt{3}} \tilde{\Gamma}_{3 \mu}
$$


where \pm indicates the spin projection $\pm 1 / 2$.

For real photons we have $\mathbf{k} \cdot \epsilon=0$ and we choose $\mathbf{k} \cdot \mathbf{p}=0$, with $\mathbf{p}$ the incoming momentum of the s-wave state. With these restrictions we can write the space-space components of the $\tilde{\Gamma}$ vertex function as:

$$
\tilde{\Gamma}_{j i}=\delta_{j i} \Gamma_{E}+k_{j} p_{i} \Gamma_{M} .
$$

We can, without loss of generality, choose the photon to be traveling in the $\hat{z}$ direction, and it then follows that $\tilde{\Gamma}_{33}=\Gamma_{E}$.

Using the definition of $\mathrm{B}(\mathrm{E} 1)$ strength (see, e.g. [30]) we find that the B(E1) strength for this transition is related to the renormalized, irreducible vertex $\bar{\Gamma}_{E}$ by:

$$
\mathrm{B}(\mathrm{E} 1)=\frac{3}{4 \pi}\left(\frac{\Gamma_{++3}}{\omega}\right)^{2}=\frac{1}{4 \pi}\left(\frac{\bar{\Gamma}_{E}}{\omega}\right)^{2},
$$

where

$$
\bar{\Gamma}_{E} \equiv \sqrt{Z_{\sigma} Z_{\pi}} \Gamma_{E}=\frac{1}{m_{R}^{2} g_{0} g_{1}} \sqrt{\frac{-12 \pi^{2} \gamma_{0}}{r_{1}}} \Gamma_{E}
$$

at leading order.

Current conservation (64) then provides an alternative way to calculate $\Gamma_{E}$, it tells us that:

$$
\omega \Gamma_{j 0}=\mathbf{k}_{j} \Gamma_{E} .
$$

But, for $\Gamma_{j 0}$, the diagram on the right of Fig. 6 need not be considered, and so

$$
\Gamma_{j 0}(\mathbf{k}) \sim \int d^{3} r \frac{u_{1}(r)}{r} Y_{1 j}(\hat{r}) e^{i \mathbf{k} \cdot \mathbf{r}} \frac{u_{0}(r)}{r},
$$

where $u_{1}$ and $u_{0}$ are the leading-order wave functions of the s- and p-wave states, given by Eq. (6). As $|\mathbf{k}| \rightarrow 0$ Eq. (71) reduces to:

$$
\Gamma_{j 0}(\mathbf{k}) \sim \mathbf{k}_{j} \int d r r u_{1}(r) u_{0}(r),
$$

an equation in which, of course, the integral is the canonical form of the E1 matrix element.

We performed both the momentum-space calculation of $\tilde{\Gamma}_{33}=\Gamma_{E}$, and the calculation of $\Gamma_{E}$ via the co-ordinate space integral (72) (with appropriate factors). The divergences cancel in the former calculation, as expected, and the same result is obtained from either integral. Inserting the result in Eq. (68) we find:

$$
\mathrm{B}(\mathrm{E} 1)=\frac{Z_{e f f}^{2} e^{2}}{3 \pi} \frac{\gamma_{0}}{-r_{1}}\left[\frac{2 \gamma_{1}+\gamma_{0}}{\left(\gamma_{0}+\gamma_{1}\right)^{2}}\right]^{2}
$$

is the leading-order Halo EFT result with

$$
Z_{\text {eff }}=\frac{m_{R}}{M} Q_{c} \equiv f Q_{c} \approx 0.366
$$

the effective charge.

No cutoff parameter is needed in order to get a finite result for $\mathrm{B}(\mathrm{E} 1)$ : our value is finite without regularization, c.f. Ref. [29. We note that the result (73) is "universal" in the 
sense that it applies to any E1 s-to-p-wave transition in a one-neutron halo nucleus. Once $r_{1}, \gamma_{1}$, and $\gamma_{0}$ are known for a given one-neutron halo the prediction $(73)$ is accurate up to corrections of order $R_{\text {core }} / R_{\text {halo }}$.

We now have to deal with the issue that we do not have a value for $r_{1}$ that has been obtained solely from an EFT calculation and data. So here we set $r_{1}$ in order to reproduce the experimental number (2) obtained in Ref. [3]. This produces:

$$
r_{1}^{\mathrm{LO}}=-0.66 \mathrm{fm}^{-1}
$$

where we do not bother to propagate the error bars from the experiment, since NLO effects are presumably a much larger source of uncertainty. This corresponds to $a_{1}=374 \mathrm{fm}^{3}$. We note, that the value of $r_{1}$ (75) lies between the two values extracted when we adopt the value of $a_{1}$ from Ref. [2] as given and obtained $r_{1}$ using formulae of LO and NLO precision in the $R_{\text {core }} / R_{\text {halo }}$ expansion (see Sec. IIF).

Short-distance effects enter B(E1) in these NLO corrections. The B(E1) $\left(1 / 2^{+} \rightarrow 1 / 2^{-}\right)$ transition therefore cannot be predicted at NLO: a counterterm appears at that order. This can be seen either from the presence of the operator $\sim L_{E 1}^{(1 / 2)}$ in Eq. (37), or from a coordinate space argument similar to the one made in the previous section for the $1 / 2^{-}$state's charge radius. In the case of the E1 transition we have the co-ordinate space integral [2, 30]:

$$
R_{01}^{(1)} \equiv A_{0} A_{1} \int_{0}^{\infty} d r r e^{-\gamma_{1} r}\left(1+\frac{1}{\gamma_{1} r}\right) e^{-\gamma_{0} r}=2 \sqrt{-\frac{\gamma_{0}}{r_{1}}} \frac{\gamma_{0}+2 \gamma_{1}}{\left(\gamma_{0}+\gamma_{1}\right)^{2}} .
$$

Again, it is finite, and this time it has a short-distance contribution

$$
R_{01 \mid S D}^{(1)} \sim 2 \sqrt{-\frac{\gamma_{0}}{r_{1}}} R_{\text {core }}
$$

which is a fraction $\sim R_{\text {core }} / R_{\text {halo }}$ of the total.

Comparing this calculation with a shell-model treatment of ${ }^{11} \mathrm{Be}$, it is clear that one effect

which is subsumed into the NLO counterterm $L_{E 1}^{(1 / 2)}$ is the transition of a neutron from a $d_{5 / 2}$ to a $p_{3 / 2}$ orbital, with that neutron coupled to the $2^{+}$state of ${ }^{10} \mathrm{Be}$. This $2^{+}$state is $3.4 \mathrm{MeV}$ above the ${ }^{10} \mathrm{Be}$ ground state, so the dynamics associated with it takes place at distances $R_{\text {core. }}$. Hence in our EFT it can only appear in short-distance operators such as that multiplying $L_{E 1}^{(1 / 2)}$ in Eq. 37). The computation of Ref. [4] suggests that such a contribution reduces the E1 matrix element by $\sim 30 \%$, which is the anticipated size of an NLO effect when the $R_{\text {core }} / R_{\text {halo }}$ expansion is employed in the ${ }^{11}$ Be system.

There are other effects of a similar size that will affect B(E1) at NLO. Specifically, there are NLO corrections from the wave-function renormalization factors associated with the swave and p-wave fields. Both tend to increase $\mathrm{B}(\mathrm{E} 1)$ over the leading-order prediction. We choose to adjust $r_{1}$ to reproduce $\mathrm{B}(\mathrm{E} 1)\left(1 / 2^{+} \rightarrow 1 / 2^{-}\right)$already at leading order, and then rely on the NLO counterterm to cancel these NLO corrections. That this is a reasonable strategy is a testable hypothesis, since the counterterm that appears here at NLO also plays a role in Coulomb dissociation of ${ }^{11} \mathrm{Be}$. It is to that process that we now turn our attention.

\section{PHOTODISINTEGRATION OF ${ }^{11}$ BE INTO ${ }^{10}$ BE AND A NEUTRON}

In this section we consider the photodisintegration of the Beryllium-11 nucleus to a Beryllium-10 nucleus plus a neutron. In practice this process is measured using Coulomb ex- 
citation of the ${ }^{11} \mathrm{Be}$ nucleus, with the two reactions connected within the equivalent-photon approximation.

Once again, we first compute photodisintegration into the direct product of a core state of definite polarization and a neutron of definite spin. The separation into states of definite total angular momentum will be done after that calculation is complete.

There are two contributions to this process, as depicted in Fig. 7. The first diagram, denoted "LO" in the figure, corresponds to the plane-wave impulse approximation contribution. Evaluation of the relevant Feynman graph yields, for the zeroth-component of the four-current:

$$
\mathcal{M}_{0}^{(a)}=\frac{e Q_{c} g_{0} 2 m_{R}}{\gamma_{0}^{2}+\left(\mathbf{p}^{\prime}-\frac{m}{M_{n c}} \mathbf{k}\right)^{2}},
$$

where $\mathbf{p}^{\prime}$ is the relative momentum of the outgoing $n c$ pair.

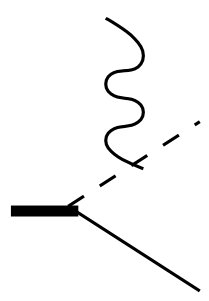

LO

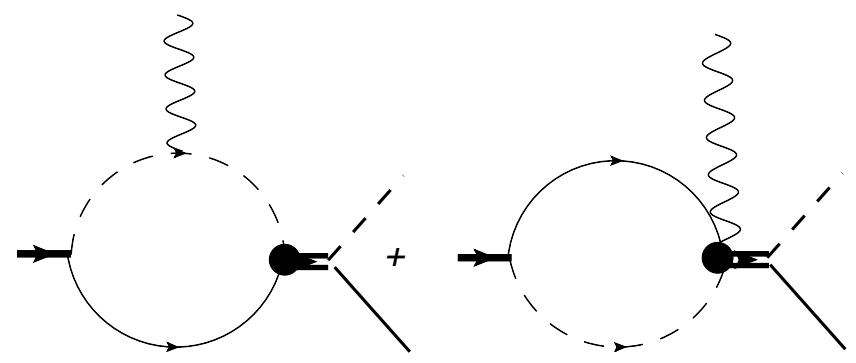

NLO

FIG. 7: The two diagrams representing photodissociation of the ${ }^{11} \mathrm{Be}$ ground (s-wave) state. As we will show below, the first diagram, denoted LO here, is dominant over diagrams involving p-wave final-state interactions.

The second diagram pair of diagrams, (b), includes final-state interactions between the neutron and the core. (In fact, these interactions are only present in the $J=1 / 2$ channel, a point we will have to deal with below.) The diagram with final-state interactions can be written as:

$$
\mathcal{M}_{0}^{(b)}=e Q_{c} g_{0}\left(2 m_{R}\right)^{2} \frac{-6 \pi}{m_{R}} \frac{1}{p^{\prime 3} \cot \delta_{1}\left(p^{\prime}\right)-i p^{\prime 3}} p_{j}^{\prime} L_{j},
$$

with $p^{\prime 3} \cot \delta_{1}\left(p^{\prime}\right)=-\frac{1}{a_{1}}+\frac{1}{2} r_{1} p^{2}$ if we do not yet expand for the case $r_{1} \sim 1 / R_{\text {core }}$. In Eq. (79) repeated indices are summed over and $L_{j}$ is the (vector) integral

$$
L_{j} \equiv \int \frac{d^{d-1} p}{(2 \pi)^{d-1}}\left(p_{j}+\frac{m}{M_{n c}} k_{j}\right) \frac{1}{p^{\prime 2}-\left(\mathbf{p}+\frac{m}{M_{n c}} \mathbf{k}\right)^{2}+i \eta} \frac{1}{\gamma_{0}^{2}+\mathbf{p}^{2}} .
$$

This can be converted into a co-ordinate space integral, with the result:

$$
L_{j}=\frac{1}{4 \pi} \hat{k}_{j} \int d r r^{2}\left(i p^{\prime}-\frac{1}{r}\right) \frac{e^{i p^{\prime} r}}{r} j_{1}\left(\frac{m}{M_{n c}} \omega r\right) \frac{e^{-\gamma_{0} r}}{r} .
$$

Thus,

$$
\mathcal{M}_{0}^{(b)}=-\frac{e Z_{e f f} g_{0} 2 m_{R} \omega}{p^{\prime 2}} \frac{e^{2 i \delta_{1}\left(p^{\prime}\right)}-1}{2 i} \hat{p}^{\prime} \cdot \hat{k} \int d r\left(i p^{\prime} r-1\right) e^{i p^{\prime} r} e^{-\gamma_{0} r}+O\left(\frac{m^{3} \omega^{3}}{M_{n c}^{3}} r^{3}\right)
$$


Meanwhile, the piece of $\mathcal{M}_{0}^{(a)}$ corresponding to a final-state $n c$ p-wave may be rewritten as:

$$
\mathcal{M}_{0}^{(a)}=e Z_{e f f} g_{0} 2 m_{R} \omega \hat{k} \cdot \hat{p}^{\prime} \int d r r^{2} j_{1}\left(p^{\prime} r\right) e^{-\gamma_{0} r} .
$$

In the presence of final-state interactions, it is most useful to write the combination $\mathcal{M}_{0}^{(a)}+$ $\mathcal{M}_{0}^{(b)}$ in the form:

$$
\mathcal{M}_{0}=e Z_{e f f} g_{0} 2 m_{R} \omega \hat{k} \cdot \hat{p}^{\prime} e^{i \delta_{1}\left(p^{\prime}\right)} \int d r r^{2}\left[j_{1}\left(p^{\prime} r\right) \cos \delta_{1}\left(p^{\prime}\right)+n_{1}\left(p^{\prime} r\right) \sin \delta_{1}\left(p^{\prime}\right)\right] e^{-\gamma_{0} r} .
$$

Note that the sign of the spherical Neumann function used here is opposite to that chosen in many textbooks. We define:

$$
n_{1}(x)=\frac{\cos (x)}{x^{2}}+\frac{\sin (x)}{x} .
$$

Next we follow Typel and Baur in Refs. 2, 30] and re-express Eq. (84) in terms of dimensionless integrals:

$$
\mathcal{M}_{0}=\frac{e Z_{e f f} g_{0} 2 m_{R} \omega}{\gamma_{0}^{3}} \hat{k} \cdot \hat{p}^{\prime} e^{i \delta_{1}\left(p^{\prime}\right)}\left[-f_{1}(y) \cos \left(\delta_{1}\left(p^{\prime}\right)\right)+f_{2}(y) \sin \left(\delta_{1}\left(p^{\prime}\right)\right)\right],
$$

where $y=p^{\prime} / \gamma_{0}$, and

$$
\begin{aligned}
& f_{1}(y) \equiv-\int_{0}^{\infty} d x x^{2} e^{-x} j_{1}(y x)=-\frac{2 y}{\left(1+y^{2}\right)^{2}}, \\
& f_{2}(y) \equiv \int_{0}^{\infty} d x x^{2} e^{-x} n_{1}(y x)=\frac{1+3 y^{2}}{\left(1+y^{2}\right)^{2} y^{2}} .
\end{aligned}
$$

This calculation corresponds to the insertion of a plane-wave for the electromagnetic field, followed by an expansion of the effects to first order in $\mathbf{r}$. The resulting matrix element differs from that of the dipole operator $|\mathbf{r}| Y_{10}(\hat{r})$ by a factor of $\sqrt{3 /(4 \pi)}$ and a $1 / \omega$. Thus to get the physical matrix element of the E1 strength $\mathcal{M}_{E 1}$ we multiply $\mathcal{M}_{0}$ by $\sqrt{3 /(4 \pi)}$ and divide by $\omega$. We must also multiply by the field renormalization $\sqrt{Z_{\sigma}}$, for the initial state. This also justifies the factors appearing in Eq. (68).

This produces the E1 coupling to the physical $\sigma$ field of a plane wave, in the absence of neutron spin, $\overline{\mathcal{M}}_{E 1}$. To get the result for a final state of good total angular momentum we need to couple $\overline{\mathcal{M}}_{E 1}$ to the neutron spinor, and then form states of good total $J$. This leads to two different $\overline{\mathcal{M}}_{E 1}$ amplitudes, one for $J=1 / 2$ and one for $J=3 / 2$.

Only the $J=1 / 2$ amplitude has final-state interaction effects. Thus there we have:

$$
\overline{\mathcal{M}}_{E 1}^{(1 / 2)}=A_{0} \frac{e Z_{e f f} \sqrt{3}}{\gamma_{0}^{3}} e^{i \delta_{(1 / 2)}\left(p^{\prime}\right)} \frac{2 y^{3} \cos \left(\delta_{(1 / 2)}\left(p^{\prime}\right)\right)+\left(1+3 y^{2}\right) \sin \left(\delta_{(1 / 2)}\left(p^{\prime}\right)\right)}{y^{2}\left(1+y^{2}\right)^{2}} \frac{1}{\sqrt{3}},
$$

where the irrelevant spin-dependent phase in the Clebsch-Gordan coefficient has been dropped. Here we have chosen the photon momentum $\hat{k}$ to be in the z-direction. The $Y_{10}\left(\hat{p}^{\prime}\right)$ that then occurs in $\mathcal{M}_{0}$ is coupled to the final-state neutron spin to produce a $\mathcal{Y}_{\left(\frac{1}{2} 1\right) \frac{1}{2} s}\left(\hat{p}^{\prime}\right)$, where $s$ is the spin projection of the initial state. The coupling of $Y_{10}\left(\hat{p}^{\prime}\right)$ to the neutron 
spinor yields the Clebsch-Gordon coefficient $\left(\frac{1}{2} s 10 \mid\left(\frac{1}{2} 1\right) \frac{1}{2} s\right)=\sqrt{\frac{1}{3}}$. The projection onto the $J=1 / 2$ continuum $n c$ state then removes this $\mathcal{Y}$. Note that physically the final-state spin projection of the core-neutron state is equal to that of the initial state because the $m_{s}$ of the neutron is not changed by the electric transition. Meanwhile, the angular-momentum projection of the nuclear state is unaffected by the photon, since it is taken to be in the z-direction. Finally, the final-state strong interactions conserve the sum $m_{s}+m_{l}$, and so $m_{j}^{\prime}$, the angular-momentum projection of the final state is equal to $s$, the spin projection of the neutron in the initial state.

For the spin-3/2 part final-state interactions are natural, and so are suppressed by three powers of $R_{\text {core }} / R_{\text {halo }}$. Thus in this piece of the amplitude we neglect diagram (b), which is equivalent to taking $\delta_{1} \rightarrow 0$ in Eq. (86). Alternatively, we can project out the E1, $J=3 / 2$, piece of the result from Eq. 78 for $\mathcal{M}_{0}^{(a)}$, and multiply by $\sqrt{Z_{\sigma}}$ to find:

$$
\overline{\mathcal{M}}_{E 1}^{(3 / 2)}=A_{0} e Z_{e f f} \sqrt{3} \frac{2 p^{\prime}}{\left(p^{\prime 2}+\gamma_{0}^{2}\right)^{2}} \sqrt{\frac{2}{3}},
$$

where an irrelevant spin-dependent phase again has been dropped. In this case the coupling of $Y_{10}\left(\hat{p}^{\prime}\right)$ to the neutron spinor yields a Clebsch-Gordan coefficient $\left(\frac{1}{2} s 10 \mid\left(\frac{1}{2} 1\right) \frac{3}{2} s\right)=\sqrt{\frac{2}{3}}$. Finally we convert to the physical observable $\frac{\mathrm{dB}(\mathrm{E} 1)}{d E}$, by:

$$
\mathrm{dB}(\mathrm{E} 1)=\left(\left|\overline{\mathcal{M}}_{E 1}^{(1 / 2)}\right|^{2}+\left|\overline{\mathcal{M}}_{E 1}^{(3 / 2)}\right|^{2}\right) \frac{d^{3} p^{\prime}}{(2 \pi)^{3}} .
$$

Inserting Eqs. (89) and (90) in Eq. (91) produces:

$$
\frac{d \mathrm{~B}(\mathrm{E} 1)}{d E}=e^{2} Z_{e f f}^{2} \frac{m_{R}}{2 \pi^{2}} A_{0}^{2}\left(\frac{\left[2 p^{\prime 3} \cos \left(\delta_{(1 / 2)}\left(p^{\prime}\right)\right)+\left(\gamma_{0}^{3}+3 \gamma_{0} p^{2}\right) \sin \left(\delta_{(1 / 2)}\left(p^{\prime}\right)\right)\right]^{2}}{p^{\prime 3}\left(p^{\prime 2}+\gamma_{0}^{2}\right)^{4}}+\frac{8 p^{\prime 3}}{\left(p^{\prime 2}+\gamma_{0}^{2}\right)^{4}}\right),
$$

where $E=p^{2} /\left(2 m_{R}\right)$ is the kinetic energy of the neutron and ${ }^{10} \mathrm{Be}$ in the center-of-mass frame. This expression can be rendered more straightforward to work with if we use the identity $\cot ^{2} \delta+1=\sin ^{-2} \delta$ to write things in terms of $p^{\prime 3} \cot \delta_{(1 / 2)}\left(p^{\prime}\right)$ :

$$
\frac{d \mathrm{~B}(\mathrm{E} 1)}{d E}=e^{2} Z_{\text {eff }}^{2} \frac{m_{R}}{2 \pi^{2}} A_{0}^{2}\left(\frac{p^{\prime 3}\left[2 p^{\prime 3} \cot \left(\delta_{(1 / 2)}\left(p^{\prime}\right)\right)+\gamma_{0}^{3}+3 \gamma_{0} p^{2}\right]^{2}}{\left[p^{\prime 6}+p^{\prime 6} \cot ^{2}\left(\delta_{(1 / 2)}\left(p^{\prime}\right)\right)\right]\left(p^{\prime 2}+\gamma_{0}^{2}\right)^{4}}+\frac{8 p^{\prime 3}}{\left(p^{\prime 2}+\gamma_{0}^{2}\right)^{4}}\right),
$$

which makes it clear that the first term (the contribution of the $J=1 / 2$ channels) does not diverge as $p^{\prime} \rightarrow 0$.

The expression (93) is true to all orders in final-state interactions, and for any value of the s-wave asymptotic normalization $A_{0}$. However, as explained above, in the ${ }^{11} \mathrm{Be}$ system, final-state interactions - even in the $J=1 / 2$ channel-are weak, and can be considered sub-leading. Also, $A_{0}=\sqrt{2 \gamma_{0}}$ at leading order, but then receives higher-order corrections in the case of a non-zero $r_{0}$. Thus we now provide order-by-order expressions for $d \mathrm{~B}(\mathrm{E} 1) / \mathrm{dE}$ in the $R_{\text {core }} / R_{\text {halo }}$ expansion.

First, at LO we have $\delta_{(1 / 2)}\left(p^{\prime}\right)=0$, and $A_{0}=\sqrt{2 \gamma_{0}}$, so:

$$
{\frac{d \mathrm{~B}(\mathrm{E} 1)^{\mathrm{LO}}}{d E}}^{\mathrm{LO}}=e^{2} Z_{\text {eff }}^{2} \frac{3 m_{R}}{2 \pi^{2}} \frac{8 \gamma_{0} p^{\prime 3}}{\left(p^{\prime 2}+\gamma_{0}^{2}\right)^{4}} .
$$


The NLO correction comes from two sources. The first is the shift of $A_{0}$ to larger values due to a $r_{0}>0$, which tends to increase the B(E1) strength. Second, final-state interactions make $\delta_{(1 / 2)}\left(p^{\prime}\right) \neq 0$. Employing Eq. (32) for $\delta_{(1 / 2)}\left(p^{\prime}\right)$ in Eq. (92) and keeping only terms up to first order in that phase shift, the total NLO result becomes:

$$
\begin{aligned}
\frac{d \mathrm{~B}(\mathrm{E} 1)}{d E}^{\mathrm{NLO}} & =e^{2} Z_{\text {eff }}^{2} \frac{3 m_{R}}{2 \pi^{2}} \frac{8 \gamma_{0} p^{\prime 3}}{\left(p^{\prime 2}+\gamma_{0}^{2}\right)^{4}}\left(\frac{A_{0}^{2}}{2 \gamma_{0}}+\frac{2 \gamma_{0}}{3 r_{1}} \frac{\gamma_{0}^{2}+3 p^{\prime 2}}{p^{\prime 2}+\gamma_{1}^{2}}\right) \\
& =e^{2} Z_{\text {eff }}^{2} \frac{3 m_{R}}{2 \pi^{2}} \frac{8 \gamma_{0} p^{\prime 3}}{\left(p^{\prime 2}+\gamma_{0}^{2}\right)^{4}}\left(1+r_{0} \gamma_{0}+\frac{2 \gamma_{0}}{3 r_{1}} \frac{\gamma_{0}^{2}+3 p^{\prime 2}}{p^{\prime 2}+\gamma_{1}^{2}}\right)
\end{aligned}
$$

where in the second line we have used (18) and kept only the term of $O\left(r_{0} \gamma_{0}\right)$. Accurate measurements of the Coulomb dissociation spectrum therefore provide information on the s-wave ${ }^{10} \mathrm{Be}$ effective range, if the p-wave effective range is already fixed from another observable.

This sort of expansion of Eq. (93) can be carried to arbitrarily high orders in $R_{\text {core }} / R_{\text {halo }}$. However, it must be remembered that the counterterm that affects $\mathrm{B}(\mathrm{E} 1)\left(1 / 2^{+} \rightarrow 1 / 2^{-}\right)$ will appear in $d \mathrm{~B}(\mathrm{E} 1) / d E$ at $O\left(R_{\text {core }}^{2} / R_{\text {halo }}^{2}\right)$, and so the expansion of FSI and asymptoticnormalization effects in powers of the EFT small parameter does not capture all of the physics of the $\mathrm{B}(\mathrm{E} 1)$ transition.

We do, however, have the advantage that the same counterterm enters both the boundto-bound and bound-to-continuum transition, so the number of free parameters needed to describe $\mathrm{B}(\mathrm{E} 1)$ strength is limited. Up to NLO accuracy for the bound-to-bound, and NNLO for the bound-to-continuum, there are five numbers: $\gamma_{0}$ and $\gamma_{1}$ (which are known from separation energies), and $r_{1}, A_{0}$, and the counterterm, which must be fitted to the available data on $\mathrm{B}(\mathrm{E} 1)\left(1 / 2^{+} \rightarrow 1 / 2^{-}\right)$and $d \mathrm{~B}(\mathrm{E} 1) / d E$.

The quality of the Coulomb excitation data is such that realistically we can hope only to extract one parameter from it, and so an NNLO analysis is not feasible at this time. In the next section we present our numerical results from the NLO analysis of the Coulomb excitation data. At NLO we need only $\gamma_{0}, \gamma_{1}, r_{1}$, and $A_{0}$ (or, equivalently, $r_{0}$ ).

\section{RESULTS FOR COULOMB EXCITATION OF THE ${ }^{11}$ BE NUCLEUS}

The data set we use is that of Ref. [5]. The predictions obtained in the previous section must be folded with the neutron detector resolution as described in that paper. For the number of E1 photons as a function of the photon energy $\omega$ we take [31]

$$
N_{E 1}(\omega)=\frac{2 Z^{2} \alpha}{\pi v^{2}}\left[\xi K_{0}(\xi) K_{1}(\xi)-\frac{v^{2}}{2} \xi^{2}\left[K_{1}^{2}(\xi)-K_{0}^{2}(\xi)\right]\right],
$$

where $\xi=\omega b /(\gamma v)$, with $b$ the impact parameter, and $\gamma=\left(1-v^{2} / c^{2}\right)^{-1 / 2}$ the Lorentz factor of the beam. We adopt the value $b=10.38 \mathrm{fm}$ and obtain $\gamma$ from the kinetic energy of the ${ }^{11}$ Be beam of $520 \mathrm{MeV}$ per nucleon, both based on values quoted in Ref. [5].

The leading-order result (94) can then be folded with detector response and the spectrum of E1 photons. This produces the red-dashed curve in Fig. 8. At next-to-leading order we take the value of $r_{1}$ fixed as per Eq. (75). We then have one free parameter, the value of $A_{0}$ at NLO (or, equivalently $r_{0}$ ) [32]. After folding we find a reasonable fit for $A_{0} / A_{0}^{\mathrm{LO}}=1.3$, which corresponds to $r_{0}=2.7 \mathrm{fm}$. The green dotted line shows the result of Typel and Baur of Ref. [30]. That result was obtained with all integrals regulated at a scale of $R=2.78$ 


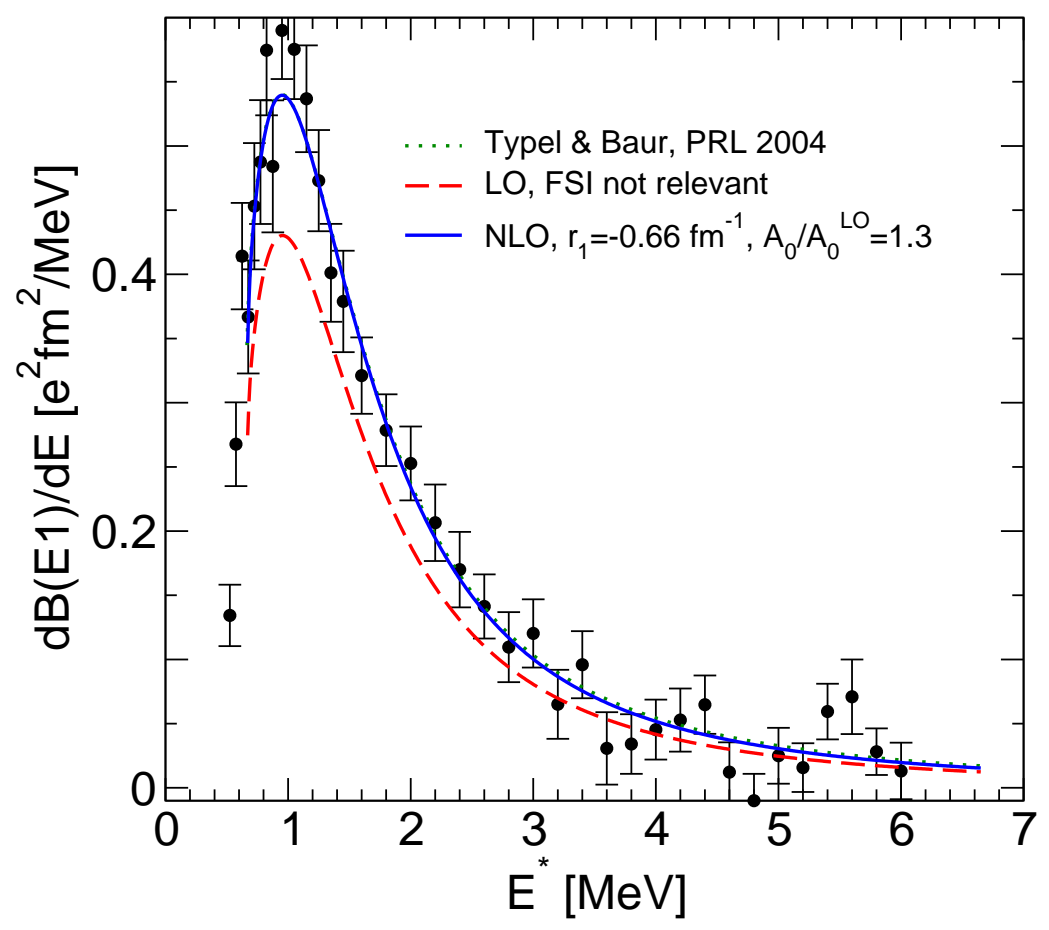

FIG. 8: Differential B(E1) strength for Coulomb dissociation of Beryllium-11 into a neutron and a ${ }^{10} \mathrm{Be}$ nucleus, plotted versus the excess energy of the detected neutron $E^{*}$, in $\mathrm{MeV}$. The data are from Ref. [5]. The theory curves have been folded with detector resolution. The red dashed line is the leading-order Halo EFT prediction, which does not include any final-state interactions. Final-state interactions, with the effective range taking on a value fixed from the bound-to-bound E1 transition strength, are included in the NLO result, which is shown in blue. The result of Ref. [30] is the green dotted line, which essentially matches the solid blue line.

fm. This corresponds to specific assumptions about all the counterterms that appear in the theory.

Note that $A_{0} / A_{0}^{\mathrm{LO}}=1.3$ increases the charge radius of the ${ }^{11} \mathrm{Be}$ ground state to

$$
\left\langle r_{E}^{2}\right\rangle_{11 \mathrm{Be}}^{(\sigma)}=2.42 \mathrm{fm}
$$

which is certainly in agreement with the atomic-physics number (5) within the expected size of NNLO corrections. This must be taken with a grain of salt, though, since the change in 
the $A=10$ to $A=11$ radius difference from LO to NLO is $\sim 30 \%$. But, in contrast to observables involving $\mathrm{p}$-wave binding, the radius of the ${ }^{11} \mathrm{Be}$ ground state does not receive any corrections from short-distance physics until $\mathrm{N}^{3} \mathrm{LO}$. The remaining difference between our NLO number (98) and the experimental number is certainly consistent with the presence of the short-distance operator $\sim L_{C 0}^{(\sigma)}$ at $\mathrm{N}^{3} \mathrm{LO}$ in the EFT expansion for the radius.

The neutron radius of the ground state of ${ }^{11} \mathrm{Be}$ is similarly increased by the presence of an $r_{0}>0$. The value of $A_{0}$ extracted from the Coulomb dissociation data produces $\left\langle r_{n}^{2}\right\rangle^{1 / 2}=5.6 \mathrm{fm}$ within the context of our NLO calculation, a $30 \%$ shift from the LO result. As with $\left\langle r_{E}^{2}\right\rangle$, the next contribution is from purely short-distance physics, and assuming that this gives a contribution of order $R_{\text {core }}$ to $\left\langle r_{n}^{2}\right\rangle^{1 / 2}$, we have the Halo EFT determination:

$$
\left\langle r_{n}^{2}\right\rangle^{1 / 2}=(5.6 \pm 0.6) \mathrm{fm}
$$

where the error bar does not account for the statistical uncertainty in the extraction of $A_{0}$. Eq. (99) is a model-independent result for the neutron radius of ${ }^{11} \mathrm{Be}$, obtained from the Coulomb dissociation data of Ref. [5].

\section{CORRELATIONS BETWEEN OBSERVABLES IN THE ${ }^{11}$ BE SYSTEM}

Effective field theories in general, and Halo EFT in particular, provide model-independent correlations between different observables. In the previous sections, we have expressed the electromagnetic properties of the ${ }^{11} \mathrm{Be}$ system through the effective range parameters for n- ${ }^{10}$ Be scattering, $\gamma_{0}, \gamma_{1}, r_{0}$, and $r_{1}$. Our expressions thus can be interpreted as correlations between scattering observables and electromagnetic properties. However, depending on the experimental information available, it could be useful to look at correlations between electromagnetic observables.

As an example, we consider the correlation between the $\mathrm{B}(\mathrm{E} 1)$ strength and the radius of the p-wave state in ${ }^{11} \mathrm{Be}$ at LO. Using Eqs. (58, 73) we obtain

$$
\mathrm{B}(\mathrm{E} 1)=\frac{2 e^{2} Q_{c}^{2}}{15 \pi}\left\langle r_{c}^{2}\right\rangle^{(\pi)} x\left[\frac{1+2 x}{(1+x)^{2}}\right]^{2},
$$

where $x=\sqrt{B_{1} / B_{0}}$ is the ratio of the neutron separation energies for the $\mathrm{p}$-wave and s-wave states. The $\mathrm{B}(\mathrm{E} 1)$ strength is thus proportional to the mean-square radius of the p-wave state. In the limit of vanishing neutron separation energy for the p-wave state, the B(E1) strength vanishes linearly with $x$. Eq. (100) can also be used to obtain the electric radius of the p-wave state $\left\langle r_{E}^{2}\right\rangle^{(\pi)}$ directly from the measured value of $\mathrm{B}(\mathrm{E} 1)$ and the neutron separation energies $B_{1}$ and $B_{0}$. This gives a radius of the ${ }^{11} \mathrm{Be} \mathrm{p}$-wave state, relative to the ${ }^{10}$ Be ground state:

$$
\left\langle r_{E}^{2}\right\rangle^{(\pi)}=0.35 \ldots 0.39 \mathrm{fm}^{2},
$$

depending on whether the value for B(E1) from Eq. (2) or Eq. (3) is used.

Analogously, the strength for the E1 transition to the continuum can be related to the radius of the s-wave state. However, this observable is also affected by the magnitude of p-wave final-state interactions. At next-to-leading order we have:

$$
{\frac{d \mathrm{~B}(\mathrm{E} 1)^{\mathrm{NLO}}}{d E}}^{\mathrm{N}}=\frac{12}{\pi^{2} B_{0}} \frac{y^{3}}{\left(1+y^{2}\right)^{4}}\left[e^{2} Q_{c}^{2} \Delta\left\langle r_{E}^{2}\right\rangle^{(\sigma)}-\pi \mathrm{B}(\mathrm{E} 1) \frac{(1+x)^{4}\left(1+3 y^{2}\right)}{\left(y^{2}+x^{2}\right)(1+2 x)^{2}}\right]
$$


where $y=p^{\prime} / \gamma_{0}$. Similar correlations can be derived for other observables and/or at higher orders. They have proven useful in the analysis of universal properties in ultracold atoms and also show promise for halo nuclei [33].

\section{CONCLUSION}

This discussion of electromagnetic observables in the Beryllium-11 system already displays the significant recent experimental activity that has been focused on this system. Coulomb excitation has been used at a variety of facilities to probe E1 transitions, and atomic-physics experiments have made great strides through advances in trapping technology. This means that the time is ripe for a detailed analysis of electromagnetic properties of halo systems. Here we have shown how EFT can provide such an analysis.

The Halo EFT we employed is complementary to ab initio methods [10-12], which can struggle to describe E1 transitions and radii in these extended nuclei because of the widely varying core and halo scales that are present in the problem. In Halo EFT this wide separation of scales is the basis for the calculation. Input that summarizes the physics at scale $R_{\text {core }}$ can be taken from either simulation or experiment, and the EFT is then used to predict the outcome of experiments that probe dynamics at the halo scale.

The results derived here in EFT mirror elegant analytic approaches to halo nuclei (see, e.g. Refs. [2, 29, 30]). But, in contrast to those works, there is no regulator dependence in our result for the E1 strength. This is a consequence of current conservation in our formalism. Moreover, the EFT delineates the order at which any observable receives a contribution from physics at scale $R_{\text {core }}$ - a contribution which cannot be calculated using the asymptotic wave functions (6). The EFT also explicitly shows whether - and if so, where - these shortdistance effects appear in other observables. In this way we can systematically assess the impact of physics at scale $R_{\text {core }}$ on low-energy electromagnetic observables in halo nuclei, and so go beyond the calculations of Refs. [2, 29, 30].

We determined the magnitude of the s-wave $\mathrm{n}^{10} \mathrm{Be}$ effective range, $r_{0}$, by examining experimental results for the low-energy E1 strength function in breakup to the ${ }^{10} \mathrm{Be}$-neutron channel: $d \mathrm{~B}(\mathrm{E} 1) / d E$. We find a reasonable fit for $r_{0}=2.7 \mathrm{fm}$. We were also able to extract a value for the $\mathrm{p}$-wave effective range $r_{1}$ from the bound-to-bound $\mathrm{B}(\mathrm{E} 1)$ strength, obtaining $r_{1}=-0.66 \mathrm{fm}^{-1}$ up to the $40 \%$ corrections which seem typical in this EFT. This gives a p-wave scattering volume $a_{1}=(374 \pm 150) \mathrm{fm}^{3}$. This overlaps the range quoted in Ref. [30], but has a lower central value. We would caution against any strong conclusion regarding values of $a_{1}$ and $r_{1}$ until the counterterm that parameterizes the short-distance piece of the E1 strength in this system has been determined by further measurements.

There are no spectroscopic factors in our approach. Nevertheless, a comparison of the values we have obtained for the physical observables $r_{0}, r_{1}$ and $a_{1}$ (or, equivalently, $A_{0}$ and $A_{1}$ ) with those found within many-body models and $a b$ initio calculations would be interesting. While the interpretation of these quantities in terms of the many-body dynamics of the $A=11$ system is not one of the goals of this work, such an interpretation could inform which observables are interesting for future investigation within the EFT, and, in particular, which ones will most reward study at higher EFT orders.

In this vein, we note that a higher-order calculation of the $\mathrm{E} 1$ observables considered here would necessitate determination of the E1 counterterm that occurs at NLO in the boundto-bound $\mathrm{B}(\mathrm{E} 1)$ strength. Better data on Coulomb dissociation of ${ }^{11} \mathrm{Be}$ at low energies would be very helpful in this regard, since the same counterterm occurs in the continuum 
E1 strength at next-to-next-to-leading order. With this one additional parameter we should be able to perform calculations to $5 \%$ or better for $d \mathrm{~B}(\mathrm{E} 1) / d E$. It is, though, important to note that the EFT used here breaks down at energies of order a few $\mathrm{MeV}$, and so the window in which the theory will give such an accurate description of data is quite limited.

But, already at the order to which we have worked here, the extraction of parameters for $n-{ }^{10} \mathrm{Be}$ scattering facilitates predictions for the electric and neutron radii of ${ }^{11} \mathrm{Be}$. We find agreement at the level expected of our NLO calculation between our result for $\left\langle r_{E}^{2}\right\rangle_{11}^{1 / 2}$ and the measurement of Ref. [8]. We also predict that the excited state of ${ }^{11} \mathrm{Be}$ has an electric radius of $2.43 \pm 0.1 \mathrm{fm}$. Meanwhile, the neutron radius of the ${ }^{11}$ Be ground state is found to be $\left\langle r_{n}^{2}\right\rangle^{1 / 2}=5.6 \pm 0.6 \mathrm{fm}$. We note that EFT provides a way to extract this quantity from the Coulomb dissociation data which is independent of any assumptions about the details of the physics that occurs at distances $\sim R_{\text {halo }}$.

Lastly, we emphasize that the Halo EFT can be used to derive correlations driven by the separation of scales in the system. These correlations may not be obvious in ab initio approaches where this separation is not explicit in the parameters of the theory. EFT has been successful at revealing these patterns in few-nucleon systems, e.g. the correlation between the triton binding energy and the spin-doublet neutron-deuteron scattering length (the "Phillips line" [34]), and the correlation between the triton and alpha particle binding energies (the "Tjon line" 35]). We now understand that those correlations are driven by the hierarchy between NN scattering lengths and the range of the NN force: $1 / a_{s}, 1 / a_{t} \ll$ $m_{\pi}$, and so they can both be derived within an appropriate EFT [36, 37]. Here we have presented analogous correlations for electromagnetic observables in the ${ }^{11}$ Be system. Similar relations have proven of significant interest in the field of ultracold atoms [33]. Future tests of correlations such as Eqs. (101) and (102) can reveal the extent to which the physics of ${ }^{11} \mathrm{Be}$, and that of other halo nuclei, is driven by "universality".

The application of the Halo EFT to other one-neutron halos, and to two-neutron halos such as ${ }^{11} \mathrm{Li}$, are obvious next steps. With the inclusion of Coulomb interactions, proton halos become accessible as well.

\section{Acknowledgments}

We are grateful to S. Typel for supplying us with the data of Ref. [5] and for assistance with the details of the convolution of theoretical results with detector resolution needed to obtain Fig. 8. We thank L. Platter for discussions regarding the Clebsch-Gordon formalism used here for the spin-1/2 and spin-3/2 fields, and C. Ji for pointing out Ref. [23] to us. This research was supported by the US Department of Energy under grant DE-FG0293ER40756, by the BMBF under contract 06BN9006, and by the Mercator programme of the Deutsche Forschungsgemeinschaft. We thank the US Institute of Nuclear Theory for its support during the program "QCD, Cold Atoms, and Few-hadron Systems", which fostered this collaboration. DRP thanks the HISKP for its hospitality, and its members for a very enjoyable time in Bonn.

[1] F. Ajzenberg-Selove and J. H. Kelley, Nucl. Phys. A 506, 1 (1990).

[2] S. Typel and G. Baur, Phys. Rev. Lett. 93 (2004) 142502. 
[3] N. C. Summers et al., Phys. Lett. B 650 (2007) 124.

[4] D. J. Millener, J. W. Olness, E. K. Warburton, and S. S. Hanna, Phys. Rev. C 28 (1983) 497.

[5] R. Palit et al. [LAND Collaboration and FRS Collaboration], Phys. Rev. C 68 (2003) 034318.

[6] N. Fukuda et al., Phys. Rev. C 70 (2004) 054606.

[7] R. Anne et al., Nucl. Phys. A 575 (1994) 125.

[8] W. Nörteshauser et al., Phys. Rev. Lett. 102 (2009) 062503.

[9] H.-W. Hammer and D. Lee, Phys. Lett. B 681 (2009) 500; H.-W. Hammer and D. Lee, arXiv:1002.4603 [nucl-th].

[10] C. Forssen, P. Navratil, W. E. Ormand and E. Caurier, Phys. Rev. C 71 (2005) 044312.

[11] C. Forssen, E. Caurier and P. Navratil, Phys. Rev. C 79 (2009) 021303.

[12] S. Quaglioni and P. Navratil, Phys. Rev. Lett. 101 (2008) 092501.

[13] D. R. Phillips and H. W. Hammer, EPJ Web Conf. 3 (2010) 06002 [arXiv:1001.1511 [nucl-th]].

[14] G. Rupak and R. Higa, arXiv:1101.0207 [nucl-th].

[15] C. A. Bertulani, H.-W. Hammer and U. Van Kolck, Nucl. Phys. A 712 (2002) 37.

[16] P. F. Bedaque, H.-W. Hammer and U. van Kolck, Phys. Lett. B 569 (2003) 159.

[17] U. van Kolck, Nucl. Phys. A 645 (1999) 273.

[18] D. B. Kaplan, M. J. Savage and M. B. Wise, Phys. Lett. B 424 (1998) 390.

[19] D. B. Kaplan, M. J. Savage and M. B. Wise, Nucl. Phys. B 534 (1998) 329.

[20] J. Gegelia, Phys. Lett. B 429 (1998) 227.

[21] M. C. Birse, J. A. McGovern and K. G. Richardson, Phys. Lett. B 464 (1999) 169.

[22] V. Pascalutsa and D. R. Phillips, Phys. Rev. C 67, 055202 (2003).

[23] J. J. Sakurai, Modern Quantum Mechanics, Addison-Wesley (1985).

[24] S. R. Beane and M. J. Savage, Nucl. Phys. A 694 (2001) 511.

[25] M. Luke and A. V. Manohar, Phys. Rev. D 55, 4129 (1997).

[26] J. W. Chen, G. Rupak and M. J. Savage, Nucl. Phys. A 653 (1999) 386.

[27] D. R. Phillips, Czech. J. Phys. 52 (2002) B49.

[28] JLab Experiment E06002, K. Kumar, P. Souder, P. Michaels, G. Uricuoli spokespersons; http://hallaweb.jlab.org/parity/prex/.

[29] S. Typel and G. Baur, Eur. Phys. J. A 38 (2008) 355.

[30] S. Typel and G. Baur, Nucl. Phys. A 759 (2005) 247.

[31] C. Bertulani, arXiv:0908.4307.

[32] D. R. Phillips, G. Rupak and M. J. Savage, Phys. Lett. B 473 (2000) 209.

[33] E. Braaten, H.-W. Hammer, Phys. Rept. 428 (2006) 259.

[34] A.C. Phillips, Nucl. Phys. A 107 (1968) 209.

[35] J.A. Tjon, Phys. Lett. B 56 (1975) 217.

[36] V. Efimov and E.G. Tkachenko, Phys. Lett. 157B (1985) 108.

[37] L. Platter, H.-W. Hammer, and U.-G. Meißner, Phys. Lett. B 607 (2005) 254. 